\title{
The health status alters the pituitary function and reproduction of mice in a Cxcr2-dependent manner
}

\author{
Colin Timaxiann,2, Isabelle Raymond-Letron ${ }^{3}$, Céline Bouclier', Linda Gulliver ${ }^{4} \mathbb{B}$, Ludovic Le Corre ${ }^{5} \mathbb{D}$, Karim Chébli ${ }^{6}$, \\ Anne Guillou ${ }^{7}$, Patrice Mollard ${ }^{7}$, Karl Balabanian ${ }^{2,8} \mathbb{C}$, Gwendal Lazennec ${ }^{1,2}$ (])
}

\begin{abstract}
Microbiota and chronic infections can affect not only immune status, but also the overall physiology of animals. Here, we report that chronic infections dramatically modify the phenotype of CxCr2 KO mice, impairing in particular, their reproduction ability. We show that exposure of Cxcr2 KO females to multiple types of chronic infections prevents their ability to cycle, reduces the development of the mammary gland and alters the morphology of the uterus due to an impairment of ovary function. Mammary gland and ovary transplantation demonstrated that the hormonal contexture was playing a crucial role in this phenomenon. This was further evidenced by alterations to circulating levels of sex steroid and pituitary hormones. By analyzing at the molecular level the mechanisms of pituitary dysfunction, we showed that in the absence of $\mathrm{Cxcr2}$, bystander infections affect leukocyte migration, adhesion, and function, as well as ion transport, synaptic function behavior, and reproduction pathways. Taken together, these data reveal that a chemokine receptor plays a direct role in pituitary function and reproduction in the context of chronic infections.
\end{abstract}

DOI 10.26508/ Isa.201900599 | Received 7 November 2019 | Revised 28 January 2020 | Accepted 29 January 2020 | Published online 10 February 2020

\section{Introduction}

Bystander chronic infections are common in rodent animal conventional facilities with a high prevalence of viruses such as mouse norovirus, parvovirus, mouse hepatitis virus, rotavirus, and bacteria such as helicobacter (Pritchett-Corning et al, 2009). Because of the possible deleterious effects of such infections, this has led to a recent trend of rethinking of the health status of animal facilities and the development of Specific and Opportunistic Pathogen-Free (SOPF) or of specific pathogen-free (SPF) animal facilities to limit the influence of the environment on the phenotype of mice, especially in the case of immune or inflammatory studies. In SOPF conditions, mice are devoid of both pathogens and opportunistic infections, whereas in SPF conditions, they are devoid of pathogens only. However, there is quite a debate about using pathogen-free mice as animal models because several reports have shown that mice exposed to bystander infections better recapitulate the human immune situation than mice housed in pathogen-free conditions (Beura et al, 2016) and that infections can affect the response to vaccination (Reese et al, 2016). Moreover, it has also been shown that transplanting C57BL/ 6 embryos into domestic wild-type mice trapped in horse stables better recapitulate human immune response than laboratory animals, reinforcing the importance of microbiota (Rosshart et al, 2019). Such pathogen-free influences could also account for some of the difficulties in translating animal studies into treatments for patients. It remains that genetic alterations produced in mouse models frequently do not lead to the same phenotypes as those observed in humans with similar alterations. One relevant example is the response to infection (Cypowyj et al, 2012). Infections are transmitted through different generations of animals, in particular during birth, but also during co-housing and breast feeding (McCafferty et al, 2013).

The role of microbiota is not only important in the context of immune studies (Hooper et al, 2012; Honda \& Littman, 2016) but also can affect the outcome of different pathologies such as inflammatory bowel disease (Bloom et al, 2011), Crohn's disease (Cadwell et al, 2010), atherosclerosis (Wang et al, 2015), arthritis (Scher et al, 2013), asthma (Thorburn et al, 2015), or cancer (Roy \& Trinchieri, 2017). Importantly, the genotype of mouse models does not contribute to the totality of phenotype observed and can be largely influenced by the various types of microbiota, within some cases, a greater impact of the microbiota than the genotype on the phenotype. This has led to the concept of "host gene plus microbe" or metagenome (Stappenbeck \& Virgin, 2016). For these reasons, the use of SPF or SOPF husbandry can be viewed as an excellent way to normalize experiments and to limit the inter-individual or interhousing variability and to improve the reproducibility of the results. However, factors other than microbiota can also deeply affect the

\footnotetext{
${ }^{1}$ Centre National de la Recherche Scientifique (CNRS), SYS2DIAG-ALCEDIAG, Cap Delta, Montpellier, France ${ }^{2}$ CNRS, Groupement de Recherche 3697 “Microenvironment of Tumor Niches," Micronit, France ${ }^{3}$ Department of Histopathology, National Veterinary School of Toulouse, France and Platform of Experimental and Compared Histopathology, STROMALab, Unité de recherche mixte (UMR) Université Paul Sabatier/CNRS 5223, Etablissement français du sang, Institut national de la santé et de la recherche médicale (Inserm) U1031, Toulouse, France ${ }^{4}$ University of Otago, Dunedin, New Zealand ${ }^{5}$ Nutrition et Toxicologie Alimentaire (NUTOX) Laboratory - INSERM Lipides, Nutrition, Cancer UMR 1231 - AgrosupDijon, Dijon, France ${ }^{6}$ Equipe Metazoan Messenger RNAs Metabolism, Montpellier, France ${ }^{7}$ Institut de Génomique Fonctionnelle, CNRS, INSERM, University of Montpellier, Montpellier, France ${ }^{8}$ Université de Paris, Institut de Recherche Saint-Louis, EMiLy, INSERM U1160, Paris, France
} 
phenotype of mouse models, including husbandry conditions, such as temperature, light-dark cycles, diet, water, noise, hygrometry, and handling of animals by care takers. Nevertheless, the microbiota is sometimes necessary to generate the phenotype. Indeed, a mouse model of Crohn's disease with mice harboring a mutation in Atg16/1 gene, showed the expected phenotype in conventional conditions but not in SPF housing (Cadwell et al, 2008, 2010). On the other hand, bystander infections can lead to a loss of a particular phenotype, such as in some models of diabetes (Bach, 2002; Okada et $\mathrm{al}, 2010$ ). This can be complicated further by the fact that the nature of microbiota can lead to different phenotypes, as exemplified in another model of Crohn's disease with mice deficient for Nod2 (Ramanan et al, 2014, 2016).

Because one of the primary effects of bystander infections will be alterations to the immune system and the inflammation process (Tao \& Reese, 2017), particular attention should be paid to proinflammatory cytokines and in particular chemokines. Chemokines are chemotactic cytokines of 60-100 amino acids that can be divided into four subtypes ( $C X C, C C, C$, or CX3C), based on the location of cysteines in the $\mathrm{N}$ terminus of the protein (Zlotnik \& Yoshie, 2000). Chemokines are ligands of seven transmembrane Gai protein-coupled receptors, signaling in particular through the phosphatidylinositol-3 kinase (PI3K)/Akt, PLC/PKC and MAPK/p38, Ras/Erk and JAK2/signal transducer, and activator of transcription (STAT3) pathways (Wang \& Knaut, 2014). Chemokines and their receptors play a major role in the trafficking of immune cells, notably during immune reaction or inflammatory events (McCully et al, 2018), but their role is not restricted to immune processes, as they have been reported to be important in a number of other physiologic or pathologic events. These include angiogenesis (Strieter et al, 2005b), metabolism (Chavey et al, 2009), chronic obstructive pulmonary disease (Henrot et al, 2019), neurodegenerative disease, and cancer (Lazennec \& Richmond, 2010; Lazennec \& Lam, 2016). Among chemokine receptors, $\mathrm{Cxcr}$, which is expressed in neutrophils and endothelial cells, appears essential in the control of angiogenesis, through the binding of $E$ (glutamate), L (leucine), R (arginine) (ELR)motif containing chemokines (CXCL1, CXCL2, CXCL3, CXCL5, CXCL6, CXCL7, and CXCL8). ELR-motif chemokines harbor the tripeptide glutamic acid-leucine-arginine motif present in the $\mathrm{N}$-terminal part of the protein (Strieter et al, 2005a). Cxcr2 regulates wound healing (Devalaraja et al, 2000), angiogenesis (Addison et al, 2000), multiple sclerosis (Liu et al, 2010), Alzheimer's disease (Tsai et al, 2002), atherosclerosis (Boisvert et al, 2000), respiratory diseases (Strieter et al, 2005b), resistance to infections (Cummings et al, 1999), and is involved in cancer (Freund et al, 2003; Ali \& Lazennec, 2007; Bieche et al, 2007; Lazennec \& Richmond, 2010). Cxcr2 KO animals exhibit splenomegaly due to an increased number of metamyelocytes and neutrophils, and impairment in the recruitment of neutrophils during acute inflammatory conditions (Cacalano et al, 1994).

Here, we report that the action of microbiota on mouse phenotype is dependent on the absence of Cxcr2 protein. In the absence of $\mathrm{Cxcr2}$, mice are clearly affected by the presence of pathogens. However, in the absence of pathogens, Cxcr2 KO mice display a similar external phenotype to that of wild-type (WT) mice in terms of their ability to reproduce and their gross appearance (Cacalano et al, 1994; Broxmeyer et al, 1996). By contrast, in conditions of bystander infections, Cxcr2 null mice exhibit an impaired reproductive ability and reduced development of reproductive organs. Using mammary gland and ovary transplant experiments, we show that reproductive function can be restored to $\mathrm{CxCr} 2 \mathrm{KO}$ mice in a WT context, despite the presence of pathogens. We also show that the absence of $\mathrm{Cxc} 2$ not only leads to susceptibility to infection but also leads to reproductive defects due to major impairment of pituitary function controlling the production of pituitary hormones. This study therefore reveals a novel role for the chemokine receptor Cxcr2 in pituitary physiology, which has been discovered in the context of microbiota infections. This has never been reported for any chemokine receptor.

\section{Results}

We have been working for a long time on Cxcr2 ligands (Freund et al, 2003, 2004; Bieche et al, 2007) and we wished to use Cxcr2 KO animals to analyze its role in vivo. Our study started with the serendipitous finding that $\mathrm{CXCr} 2 \mathrm{KO}$ animals had distinct breeding abilities in conventional or SOPF animal facilities. To evaluate the possible action of microbiota mouse phenotype in the context of Cxcr2 deficiency, mice were housed either in an SOPF animal facility in sterile conditions or in a conventional animal facility with possible bystander infections. In SOPF conditions, Cxcr2 KO animals displayed the same breeding ability as WT animals, confirming prior work of Cacalano et al (1994) (Fig 1A).
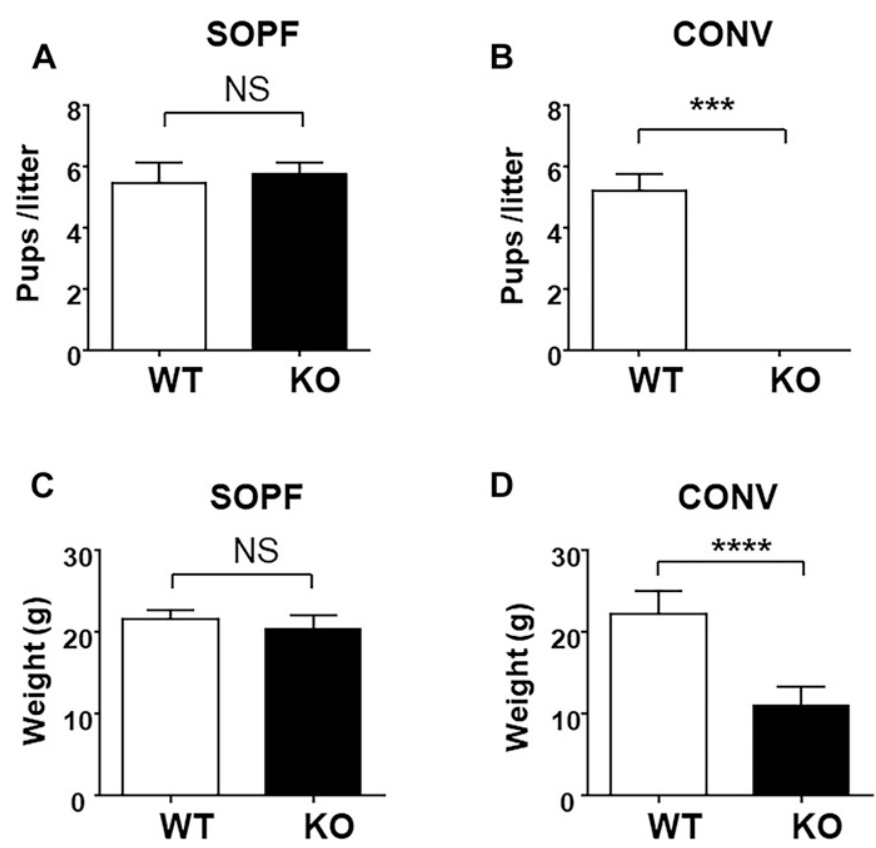

Figure 1. Husbandry in conventional housing conditions alters the reproduction of $\mathrm{CxCr} 2 \mathrm{KO}$ animals.

(A) Number of animals/litter issued in the breeding of WT animals or Cxcr2 KO animals in SOPF conditions. The data represent the mean \pm SEM of at least 10 matings (Mann-Whitney test, NS, nonsignificant, ${ }^{*} P<0.05$, ${ }^{* *} P<0.01$, ${ }^{* *} P<$ 0.001). (B) Same breeding experiment in conventional conditions with bystander infections. The results represent the mean \pm SEM of at least eight animals. (C) Weight of 12-wk-old female mice in SOPF conditions. (D) Weight of 12 -wk-old female mice in conventional conditions. Data represent the mean $\pm \mathrm{SEM}$ of at least eight animals. (Mann-Whitney test, $\left.{ }^{* * *} P<0.0001\right)$. 
On the contrary, after the transfer of SOPF animals in conventional conditions, we observed that after several generations in conventional conditions, Cxcr2 KO animals lost their breeding ability. Both males and females were infertile (Fig 1B), even when mated with WT animals. Of particular note, the products of such mating, Cxcr2 heterozygous animals, were much less affected by these conditions and could breed nearly normally (although with a delayed time for successful breeding) and generate Cxcr2 KO animals (data not shown). Moreover, Cxcr2 KO females of conventional conditions exhibited a smaller weight than WT animals (Fig 1D), which was not the case in SOPF conditions (Fig 1C). Screening of bystander infections of animals housed in conventional conditions showed the presence of mouse norovirus, helicobacter, and Entamoeba sp. (Fig S1). We hypothesized that these infections were responsible for the observed phenotype as rederivating conventional animals to remove pathogens led to animals with full reproduction ability. Moreover, this combination of pathogens was not specific for the phenotype observed, as housing Cxcr2 KO animals in other conventional facilities with another set of pathogens (including mouse norovirus, mouse hepatitis virus, other strains of helicobacter, or pinworms) led to the same results (Fig S1).

To understand why Cxcr2 KO animals were infertile in conventional conditions, we decided to focus on females. We first performed vaginal smears of WT and KO animals of conventional conditions (Fig 2A). This showed that WT animals displayed a classical cycling with proestrus, estrus, metestrus, and diestrus. On the other hand, KO animals displayed mixed populations of cells, with no real homology to any steps of estrus cycling, suggesting that the mice were not cycling. In SOPF conditions, WT and KO animals displayed a normal estrus cycle (Fig S2). To assess the functionality of the ovary, we analyzed the ovaries of WT and KO animals from conventional or SOPF conditions. We observed that in SOPF conditions, both WT and KO animals displayed a normal histology of the ovary, with all stages of follicle maturation and the presence of multiple corpora lutea, suggesting that the mice were able to ovulate (Fig 2B, upper panel). On the contrary, in conventional conditions, whereas the ovary of the WT animals appeared to have completely normal histologic appearance, ovaries from KO animals displayed a large number of follicles in all stages of development including atresia, but did not exhibit any corpus luteum, suggesting that KO Cxcr2 mice could not ovulate (Fig 2B, lower panel).

We next looked at other reproductive organs, including uterus and mammary gland. The uterus of WT and KO animals in SOPF conditions appeared normal and of similar size (Fig 3A, upper panel). In conventional conditions, the uterus of $\mathrm{KO}$ animals was smaller in diameter compared with WT animals and was in a rest status, whereas WT uterus was cycling (Fig 3A lower panel). Maximum uterine thickness and the external uterine diameter were approximately fourfold reduced in KO animals compared with WT animals (Fig S3A). Whereas uteri of WT mice had well-defined layers, uterine layers were less discernible in $\mathrm{KO}$ animals, appearing compressed and very cellular. KO mice also showed a loss of the normal convoluted appearance of the uterine luminal epithelium, assuming a more linear profile. The endometrium thickness, the luminal epithelium thickness, and the number of glandular lumen were also decreased in $\mathrm{KO}$ animals, suggesting a noncycling uterus (Fig S3B).
A
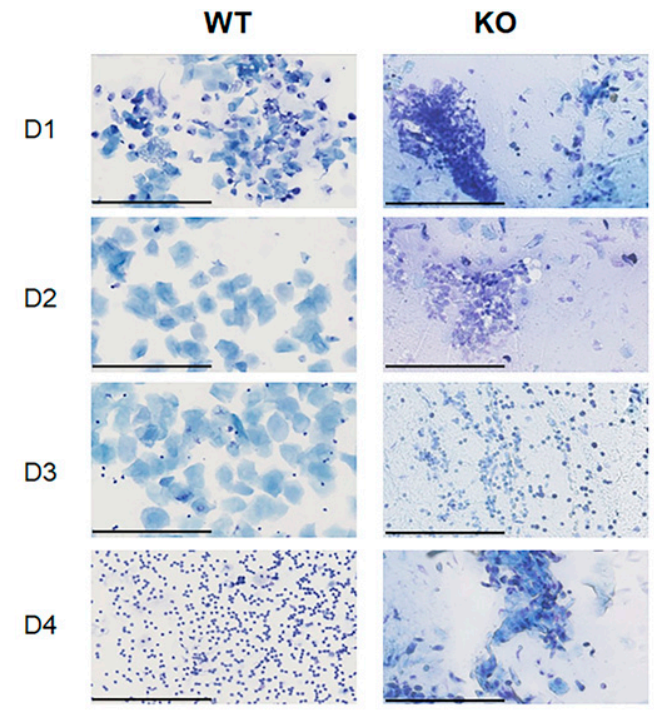

CONV
B

SOPF
CONV

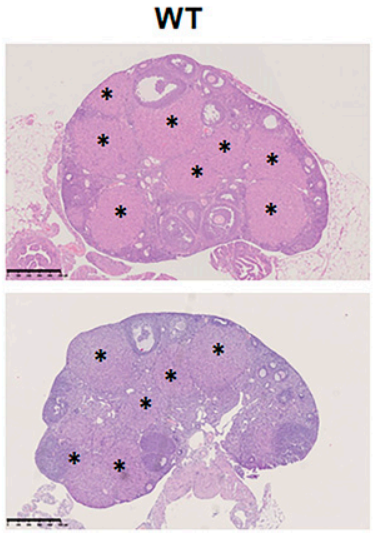

KO

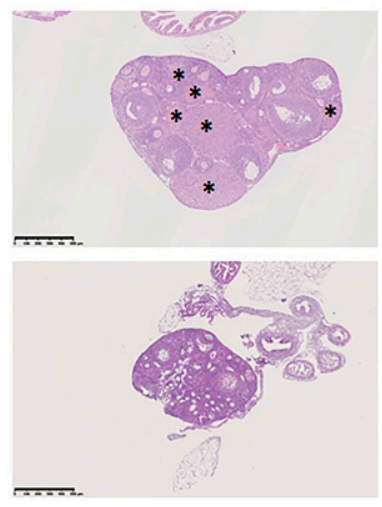

Figure 2. Cxcr2 KO animals in conventional housing conditions exhibit cycle defects and altered ovary morphology.

(A) Representative bright-field microscopic images (40×) for Giemsa-stained vaginal smears from different estrous cycle stages of WT and KO animals housed in conventional conditions. For WT animals, day 1: proestrus (mostly nucleated epithelial cells), day 2: estrus (cornified epithelial cells), day 3: metestrus (cornified epithelial cells with leukocytes), and day 4: diestrus (mostly leukocytes). For KO animals, the content of vaginal smears, no clear state of estrus cycle could be determined. Scale bars: $200 \mu \mathrm{m}$. (B) Histology of the ovary of WT and KO animals in SOPF (upper part) or conventional conditions (lower part). Representative images of hematoxylin-eosin stained ovaries at a $5 \times$ magnification are shown here. Stars indicate the presence of corpus lutea. Scale bars: $500 \mu \mathrm{m}$

We also observed an altered morphology of the mammary gland in $\mathrm{KO}$ animals in conventional conditions. Whole-mount experiments showed a complete branching in WT animals, whereas the mammary gland of $\mathrm{KO}$ animals displayed a rudimentary branching (Fig 3B). Mammary glands of $\mathrm{KO}$ appeared to have a significant reduction in the numbers of glandular (ductal) profiles (Fig S3C, lower panel). Epithelial cells lining ducts in KO mice often appeared haphazardly arranged and the mammary gland than its WT counterpart. The situation appeared different in the mammary gland of SOPF animals with a similar branching in WT and $\mathrm{KO}$ animals (Fig S3C, upper panel).

To understand the reasons for the reproductive defects in $\mathrm{KO}$ animals housed in conventional conditions, we decided to first compare the transcriptomic profiles of the mammary gland of WT 
A

WT

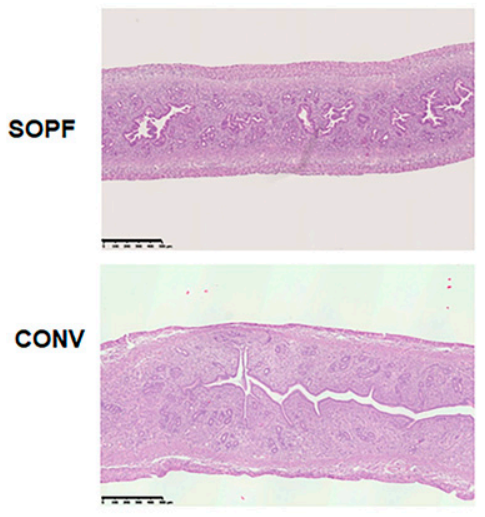

B

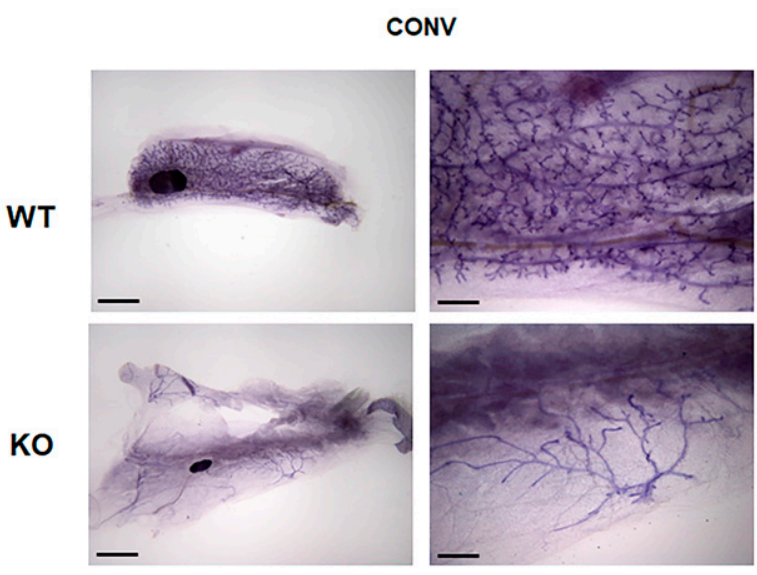

Figure 3. The uterus and mammary gland of $\mathrm{Cxcr} 2 \mathrm{KO}$ animals show defects in conventional conditions.

(A) Histology of the uterus of WT and KO animals in SOPF (upper part) or conventional conditions (lower part). Representative images of hematoxylin-eosin-stained uteri at a $5 \times$ magnification are shown here. Scale bars: $500 \mu \mathrm{m}$. (B) Whole mount of mammary glands from $13 \mathrm{wk}$ WT and KO animals in conventional conditions. Scale bars: $5 \mathrm{~mm}$ (left panel) or $1.3 \mathrm{~mm}$ (right panel).

and KO animals in SOPF with those in conventional conditions by RNAseq. Principal component analysis of RNAseq showed that mammary glands of WT and KO animals were close to each other, whereas the one of WT and KO conventional animals were more widely distributed (Fig S4). We observed that the transcriptome of the mammary gland of KO animals was much more altered in conventional than that of SOPF conditions, with $\sim 10$-fold more genes up-regulated in WT animals versus KO animals (Fig 4A). Among the genes up-regulated in the mammary gland of WT compared with $\mathrm{KO}$ animals, only 12 were common between conventional and SOPF conditions (Fig 4B), and only four were common for down-regulated genes. Gene ontology (GO) analysis showed that the common down-regulated genes were essentially related to leukocyte chemotaxis and migration, and host defense (Fig 4C). In SOPF conditions, the genes down-regulated in the mammary gland of KO animals were essentially those linked to muscle development and differentiation (Fig 4D and Table 1), whereas the genes upregulated in $\mathrm{KO}$ mice were related to granulocyte migration and leukocyte aggregation/adhesion (Fig 4E and Table 2). We next focused on the major alterations of the transcriptome of the mammary gland of KO animals in conventional conditions. GO analysis showed that most of the genes down-regulated in the mammary gland of KO animals were related to three major biological processes (Table 3): mammary gland development and differentiation (Fig 5A), epithelial cell proliferation (Fig 5B), and Wnt signaling (Fig $5 \mathrm{C}$ ). On the other hand, genes up-regulated in the mammary gland of KO animals in conventional conditions (Table 4) were involved in leukocyte migration (Fig 5D) and muscle function (Fig 5E). The RNAseq data were validated by real-time PCR on a subset of representative genes of the different $\mathrm{GO}$ identified or known to play a role in mammary gland physiology. We observed a common up-regulation of S100a8, S100a9, Mmp8, and Ngp genes related to chemotaxis in the mammary gland of $\mathrm{KO}$ animals in conventional and SOPF conditions (Fig 6). On the other hand, Areg, lactoferrin, CXCL15, Elf5, Sox10, Ido1, Wnt2, Prlr, Krt15, and Gata3 were down-regulated only in the mammary gland of $\mathrm{KO}$ animals in conventional conditions (Fig 6). Of particular note, Areg, Lactoferrin, Wnt2, Prlr, Elf5, and Gata3 are genes known to be critical for development of the mammary gland according to the studies performed with KO animals for these genes (Luetteke et al, 1999; Kelly et al, 2002; Zhou et al, 2005; Kouros-Mehr et al, 2006; Watson \& Khaled, 2008).

We also analyzed the differences in the ovary of WT and KO animals in conventional conditions, by looking at some key genes known to play a role in ovary function. We report a decrease in the expression of Akrc18, Cyp19, Hsd3b2, Prlr, and lactoferrin genes in the ovary of KO animals, whereas AR expression was strongly induced (Fig 7A). Akr1c18 encodes 20a-hydroxysteroid dehydrogenase, a progesterone-metabolizing enzyme (Piekorz et al, 2005). Cyp19 or estrogen synthase is an aromatase of the P450 family involved in, in particular, the aromatization of androgens to estrogens (Rosenfeld et al, 2001). Hsd3b2 encodes hydroxy-delta5 -steroid dehydrogenase, 3 beta-, and steroid delta-isomerase 2 , which is involved the conversion of 5 -ene-3 3 -hydroxysteroids to 4-ene-3-ketosteroid, an essential step in the biosynthesis of progesterone and estrogens in the ovary (Payne et al, 1995). Interestingly, many of these enzymes are regulated by prolactin (PLR), and prolactin receptor (Prlr) is critical (Bachelot \& Binart, 2005; Stocco et al, 2007). Androgen receptor (Ar) also plays a critical role in ovary function, and Ar KO leads to premature ovarian failure (Shiina et al, 2006; Walters, 2015). The alteration of these key regulatory genes in the ovary suggested to us a possible impairment of hormone production. We thus measured progesterone and estradiol serum levels in WT and $\mathrm{KO}$ animals in conventional conditions. In agreement with the absence of corpus luteum in KO ovaries, we observed a decrease in progesterone levels relative to WT (Fig 7B). On the other hand, estradiol levels were increased.

This led us to hypothesize that an alteration of the hormonal context could explain the reproductive defects observed in KO animals housed under conventional conditions. To test this, we performed ovary transplantation experiments (Fig 7C). WT mice were ovariectomized and a WT or KO ovary was reimplanted within the oviduct bursa (Behringer, 2017). We observed that both WT and KO transplanted ovaries were able to display a normal phenotype with the presence of corpora lutea (Fig 7 C). Moreover, when transplanted females were bred, they were able to give birth (Fig $7 C$ ). To confirm the role of the hormonal environment in the KO defects, we also performed mammary gland transplantation (Fig 7D). The 
A
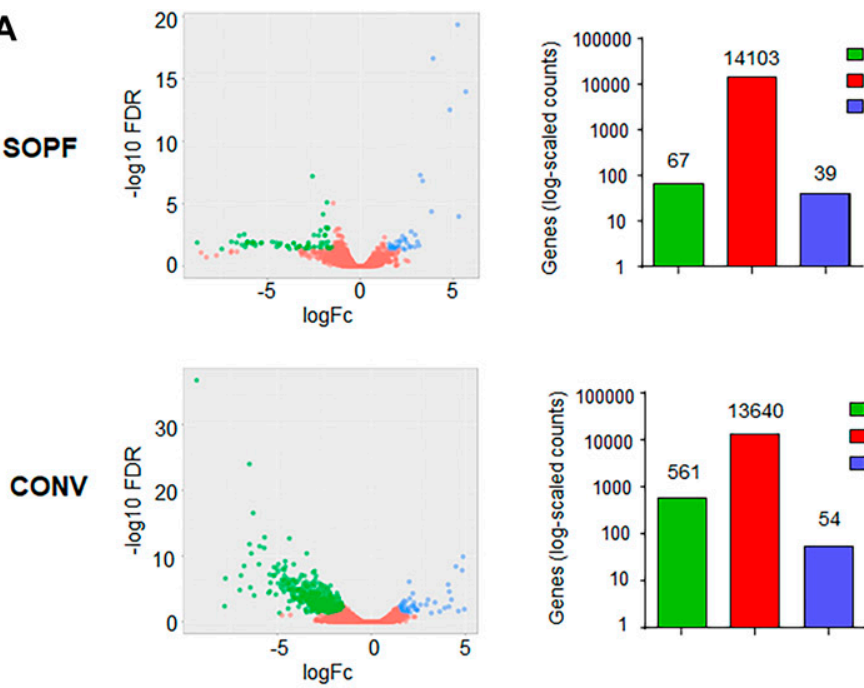

Up in WT

Not regulated

Down in WT

SOPF

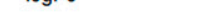

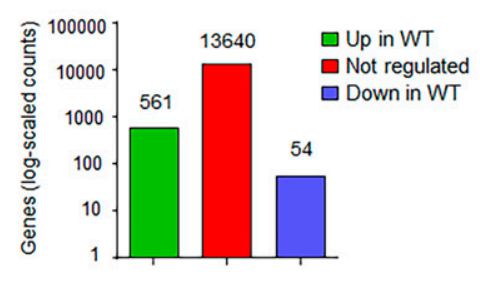

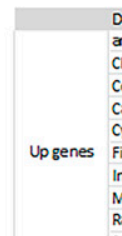

Description Descripsion Chloride intracellula channel 6 Contactin associs Caseinkappa Cytochrome $P 450$, fam Fibrinogen betachain

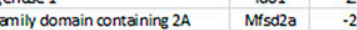

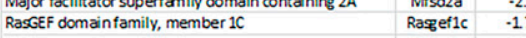
Sphingomyel in synthase 2 Segeted phosphoprotein 1 Matrix metallopeptidase 8 Neutrophilic granule protein S100 calcium binding protein $A 8$
S100 calcium binding protein A9

C

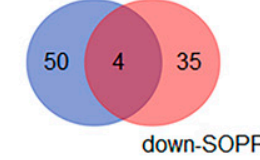

\begin{tabular}{|c|c|c|}
\hline Gene & Log FCSOPF & Log Fcconv \\
\hline Areg & -248 & -6.37 \\
\hline Clic5 & -1.88 & -4.64 \\
\hline Cntnap2 & -2.1 & -3.78 \\
\hline Csn3 & -1.92 & -4.11 \\
\hline Cyp3a57 & $-2,00$ & -4.52 \\
\hline Fgb & -221 & -4.15 \\
\hline Ido1 & -2.85 & -6.47 \\
\hline Mfsd2a & -2.1 & -3.05 \\
\hline Ragef1c & -1.74 & -3.01 \\
\hline Sgms2 & -1.73 & -1.54 \\
\hline Spp1 & -223 & -4.95 \\
\hline Mmp8 & 3.34 & 4.07 \\
\hline Ngp & 4.81 & 3.89 \\
\hline S100a3 & 3.9 & 4.87 \\
\hline S100a9 & 5.23 & 4.82 \\
\hline
\end{tabular}

Biologic Process Term Count \% PValue Genes GO:0030593 neutrophil chemotaxis GO:1990266 neutrophil migration GO:0071621 granulocyte chemotaxis GO:0097530 granulocyte migration GO:0032101 regulation of response to external stimulus GO:0097529 myeloid leukocyte migration GO:0030595 Meukocyte chemotaxis GO:0006952 defense response

D

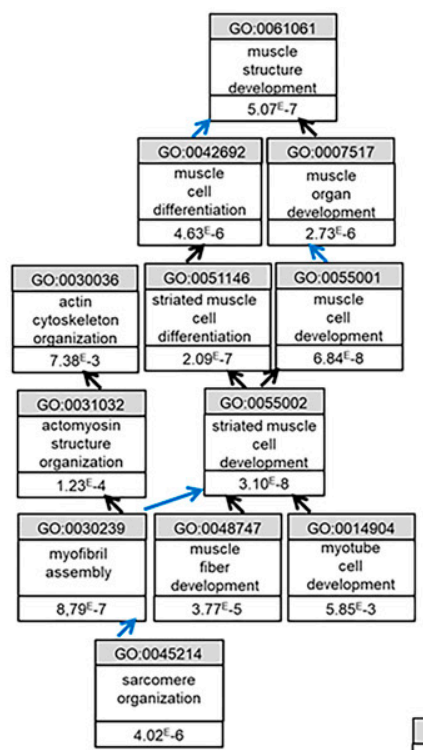

SOPF
E

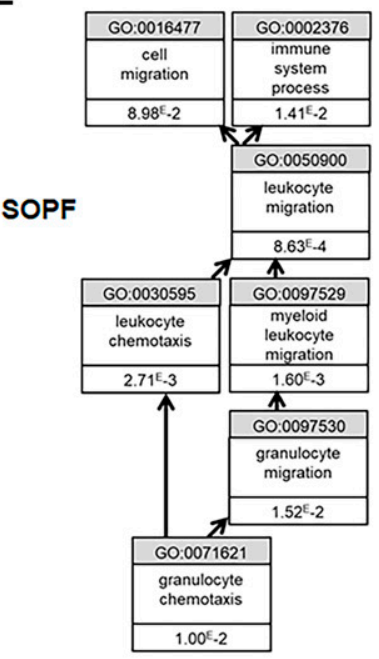

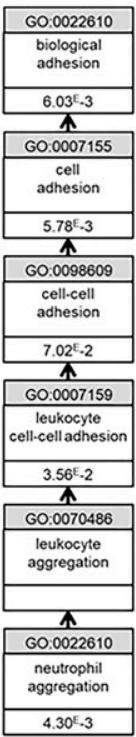

Figure 4. Differential gene expression in WT and KO mammary glands is more pronounced in conventional conditions.

(A) Left panel: the volcano plots show the global changes in RNA expression patterns for WT versus KO mammary glands in SOPF or conventional conditions. Data represent analysis of cpm estimates with a log of fold change of more than 1.5-fold change and $P<0.05$ of 3 animals per group. Right panel: number of differentially regulated genes for the same analysis. (B) Left panel: Venn diagram representing the common genes up-regulated or down-regulated in the mammary gland of WT compared with KO animals in conventional versus SOPF conditions. Right panel: list of common genes. (C) Gene Ontology (GO) analysis of biological process for the common genes regulated in $\mathrm{KO}$ animals in conventional and SOPF conditions. (D) GO analysis of biological process of down-regulated genes in KO mammary glands of SOPF is mostly related to muscle function. Black arrows mean "is a." Blue arrows mean is "part of." (E) GO analysis of biological process of up-regulated genes in KO mammary glands of SOPF are mostly related to granulocyte chemotaxis (left panel) and to neutrophil aggregation (right panel).

mammary fat pads of young WT female mice were cleared of all epithelial structures and either reimplanted with WT or KO mammary gland fragments or left untreated. Control mammary glands without transplant did not develop any ductal branching, whereas both WT and KO transplants could fully restore a functional mammary gland (Fig 7D). Together, these data suggest that the hormonal environment of WT animals is sufficient to enable the KO ovary and mammary gland to be functional. As steroid hormone production is controlled by the pituitary, we assessed the serum levels of the pituitary hormones follicle stimulating hormone (FSH), luteinizing hormone (LH), PRL, and growth hormone (GH) in WT and $\mathrm{KO}$ animals in conventional conditions. We report that the four pituitary hormones tested displayed a clear decrease in $\mathrm{KO}$ animals (Fig 8A), suggesting major defects in the pituitary function of $\mathrm{KO}$ animals housed under conventional conditions. We did not measure the hormone levels of transplanted animals (Fig 7C), as these mice were used for breeding and could not be compared with virgin animals.

To understand at the molecular level, the reasons for the pituitary dysfunction in $\mathrm{KO}$ animals, we performed an RNAseq analysis of pituitary glands from WT and KO animals in SOPF and conventional conditions (Fig 8B). Strikingly, very little difference was observed between the pituitaries of WT and KO animals in SOPF conditions. On the other hand, more than 850 genes were either up-regulated or down-regulated in the pituitary of $\mathrm{KO}$ animals in 
Table 1. Most enriched pathways for genes down-regulated in the mammary gland of KO versus WT specific and opportunistic pathogen-free animals.

\begin{tabular}{|c|c|c|c|c|}
\hline GO biological process term & Count & $\%$ & $P$-value & Genes \\
\hline GO:0003012 muscle system process & 15 & 23.43 & $3.92 \mathrm{E}-12$ & $\begin{array}{l}\text { Cmya5, Mybpc2, Tmod1, Ttn, Tcap, Srl, Atp2a1, Myl1, Hrc, Ryr1, } \\
\text { Casq1, Actn3, Tnni2, Cacna1s, and Myom1 }\end{array}$ \\
\hline GO:0006936 muscle contraction & 13 & 20.31 & $3.40 \mathrm{E}-11$ & $\begin{array}{l}\text { Mybpc2, Tmod1, Ttn, Tcap, Atp2a1, Myl1, Hrc, Ryr1, Casq1, } \\
\text { Actn3, Tnni2, Cacna1s, and Myom1 }\end{array}$ \\
\hline GO:0006941 striated muscle contraction & 9 & 14.06 & 1.60E-8 & Tnni2, Ttn, Tcap, Atp2a1, Myl1, Hrc, Casq1, Actn3, and Cacna1s \\
\hline GO:0055002 striated muscle cell development & 9 & 14.06 & $3.12 \mathrm{E}-8$ & Actn3, Tmod1, Ttn, Cacna1s, Tcap, Ldb3, Ryr1, Casq1, and Neb \\
\hline GO:0055001 muscle cell development & 9 & 14.06 & $6.84 \mathrm{E}-8$ & Actn3, Tmod1, Ttn, Cacna1s, Tcap, Ldb3, Ryr1, Casq1, and Neb \\
\hline GO:0051146 striated muscle cell differentiation & 10 & 15.62 & $2.09 \mathrm{E}-7$ & $\begin{array}{l}\text { Actn3, Tmod1, Ttn, Cacna1s, Smyd1, Tcap, Ldb3, Ryr1, Casq1, } \\
\text { and Neb }\end{array}$ \\
\hline GO:0090257 regulation of muscle system process & 9 & 14.06 & $2.37 \mathrm{E}-7$ & Actn3, Tnni2, Cmya5, Ttn, Srl, Atp2a1, Ryr1, Hrc, and Casq1 \\
\hline G0:0061061 muscle structure development & 13 & 20.31 & 5.07E-7 & $\begin{array}{l}\text { Jph1, Tmod1, Ttn, Smyd1, Tcap, Neb, Casq1, Ryr1, Actn3, } \\
\text { Cacna1s, Mylpf, Jph2, and Ldb3 }\end{array}$ \\
\hline GO:0030239 myofibril assembly & 6 & 9.37 & $8.79 \mathrm{E}-7$ & Tmod1, Ttn, Tcap, Ldb3, Casq1, and Neb \\
\hline GO:0007517 muscle organ development & 10 & 15.62 & $2.73 \mathrm{E}-6$ & $\begin{array}{l}\text { Actn3, Jph1, Ttn, Cacna1s, Jph2, Mylpf, Smyd1, Tcap, Ryr1, and } \\
\text { Casq1 }\end{array}$ \\
\hline GO:0044057 regulation of system process & 11 & 17.18 & $2.91 \mathrm{E}-6$ & $\begin{array}{l}\text { Actn3, Tnni2, Cmya5, Ttn, Fgb, Cck, Srl, Atp2a1, Ryr1, Hrc, and } \\
\text { Casq1 }\end{array}$ \\
\hline GO:0045214 sarcomere organization & 5 & 7.81 & 4.02E-6 & Ttn, Tcap, Ldb3, Casq1, and Neb \\
\hline G0:0042692 muscle cell differentiation & 10 & 15.62 & 4.63E-6 & $\begin{array}{l}\text { Actn3, Tmod1, Ttn, Cacna1s, Smyd1, Tcap, Ldb3, Ryr1, Casq1, } \\
\text { and Neb }\end{array}$ \\
\hline GO:0003009 skeletal muscle contraction & 5 & 7.81 & 5.63E-6 & Actn3, Tnni2, Tcap, Atp2a1, and Casq1 \\
\hline
\end{tabular}

conventional conditions (Fig 8B). Principal component analysis of RNAseq showed that pituitary of WT and KO animals were close to each other, whereas the one of WT and KO conventional animals were very different (Fig S4). According to GO analysis, the up-regulated pathways in the KO pituitaries were related to immune cell activation (in particular lymphocyte), leukocyte adhesion, and neutrophil motility and extravasation (Fig $8 \mathrm{C}$ and Table 5). On the other hand, downregulated genes involving biological processes included those involved in ion transport and synaptic function (Fig 8D and Table 6), as well as control of ovarian function (Fig $8 \mathrm{E}$ and Table 6). To validate these data, we analyzed the expression of a set of genes representative of the different GO mentioned above by real-time PCR on a larger number of animals (Fig 9). Elane, S100a8, S100a9, Mpo, Ngp, $M M P 8, L t f$, and Serpina3an were strongly up-regulated in the pituitary of $\mathrm{KO}$ animals in conventional conditions and modestly or not regulated at all, in the pituitary of KO animals in SOPF conditions. Elane, S100a8, and S100a9 are involved in migration, adhesion, and immune response. Mpo, Ngp, Ltf, and Serpina3an are contributing to migration and immune response. In contrast, Prl, Crhbp, Akr1c14, Vip, and Rln were all down-regulated in the pituitary of conventionally housed $\mathrm{KO}$ animals but not in SOPF conditions (Fig 9). Prl, Crhbp, and Vip are involved in ion transport, behavior and reproduction. Reln and Crhbp play a role in ion transport, synapse function and behavior.

\section{Discussion}

In this study, we investigated the effects of bystander infections on the physiologic role of the chemokine receptor Cxcr2 using Cxcr2- null mice. Our results have demonstrated that when exposed to common infections found in animal facilities, Cxcr2 KO mice, but not WT mice, exhibit reproductive defects. Of particular note, Cxcr2 ligand levels were not altered in the mammary gland and the pituitary gland of WT animals in conventional conditions compared with SOPF conditions, according to RNAseq analysis. Homozygous males and females were both sub-fertile and females displayed alterations to their secondary sex organs. The first observation accounting for this sub-fertility was that female Cxcr2 KO mice housed in conventional conditions were not able to cycle normally. None of the characteristic phases of the estrous cycle could be identified in these animals by vaginal smear, with mixed populations of vaginal cells suggesting a defect in ovarian function. Microscopic observation of ovaries from Cxcr2 KO animals housed in conventional conditions showed reductions of the size of the ovary and an absence of corpora lutea, whereas in SOPF conditions, both WT and CXcr2 KO ovaries showed normal histology with all stages of follicle development and corpora lutea present. Interestingly, a number of genes involved in ovarian function and production of steroid hormones (Akr1c18, Cyp19, Hsd3b2, Prlr, lactoferrin, and $\mathrm{Ar}$ ) were down-regulated in the ovary of $\mathrm{CxCr} 2 \mathrm{KO}$ animals in conventional conditions. Akr1c18 (20-alpha-hydroxysteroid dehydrogenase), which catabolizes progesterone into 20-alphadihydroprogesterone (inactive steroid) is necessary for the maintenance of pregnancy (Choi et al, 2008) and KO of this gene leads to longer duration of estrous cycle and a reduced number of pups (Ishida et al, 2007). Knocking down Cyp19 (Aromatase P450) has been shown to lead to mice lacking corpus luteum in ovary, accompanied by total infertility (Toda et al, 2001). Hsd3b2 (hydroxy-delta-5-steroid 
Table 2. Most enriched pathways for genes up-regulated in the mammary gland of KO versus WT specific and opportunistic pathogen-free animals.

\begin{tabular}{|c|c|c|c|c|}
\hline GO biological process term & Count & $\%$ & $P$-value & Genes \\
\hline $\begin{array}{l}\text { GO:0002523 leukocyte migration involved in inflammatory } \\
\text { response }\end{array}$ & 3 & 7.5 & $2.66 \mathrm{E}-4$ & S100a8, S100a9, and Elane \\
\hline GO:0050900 leukocyte migration & 5 & 12.5 & $8.63 \mathrm{E}-4$ & S100a8, S100a9, Elane, Thbs1, and Calca \\
\hline GO:0052547 regulation of peptidase activity & 5 & 12.5 & $1.53 \mathrm{E}-3$ & S100a8, S100a9, Thbs1, Wfdc18, and Ngp \\
\hline GO:0097529 myeloid leukocyte migration & 4 & 10.0 & $1.60 \mathrm{E}-3$ & S100a8, S100a9, Thbs1, and Calca \\
\hline GO:0030595 leukocyte chemotaxis & 4 & 10.0 & $2.71 \mathrm{E}-3$ & S100a8, S100a9, Thbs1, and Calca \\
\hline GO:0044707 single-multicellular organism process & 17 & 42.5 & $3.79 \mathrm{E}-3$ & $\begin{array}{l}\text { Col9a3, Mmp8, Mpo, Igf2, Krt10, Elane, Muc4, Slc5a1, Gjb2, } \\
\text { Thbs1, Calca, Irx4, S100a9, Mfap4, Rbp1, Ngp, and S100a8 }\end{array}$ \\
\hline GO:0006952 defense response & 8 & 20.0 & $3.99 \mathrm{E}-3$ & S100a9, Mpo, Igf2, Elane, Thbs1, Ngp, S100a8, and Calca \\
\hline GO:0070488 neutrophil aggregation & 2 & 5.0 & 4.30E-3 & S100a8 and S100a9 \\
\hline GO:0007155 cell adhesion & 8 & 20.0 & $5.78 \mathrm{E}-3$ & S100a9, Igf2, Elane, Thbs1, S100a8, Calca, Mfap4, and Muc4 \\
\hline GO:0022610 biological adhesion & 8 & 20.0 & $6.02 \mathrm{E}-3$ & S100a9, Igf2, Elane, Thbs1, S100a8, Calca, Mfap4, and Muc4 \\
\hline GO:0060326 cell chemotaxis & 4 & 10.0 & $6.04 \mathrm{E}-3$ & S100a8, S100a9, Thbs1, and Calca \\
\hline
\end{tabular}

dehydrogenase, 3 beta-, and steroid delta-isomerase 2) plays a crucial role in the biosynthesis of many steroids (Chapman et al, 2005). Prlr (prolactin receptor) KO animals are completely infertile with irregular estrous cycles (Horseman et al, 1997). Lactotransferrin, present in the reproductive tracts of rodents, can regulate secretory function and plays a role in fertilization (Yanaihara et al, 2007). Ar (androgen receptor) KO mice show a marked reduction in follicular maturation at maturity, with fewer corpora lutea in their ovaries (Hu et al, 2004). Not surprisingly then, morphometry, using multiple histological sections in the present study, identified alterations to

Table 3. Most enriched pathways for genes down-regulated in the mammary gland of KO versus WT conventional animals.

\begin{tabular}{|c|c|c|c|c|}
\hline GO biological process term & Count & $\%$ & $P$-value & Genes \\
\hline GO:0007155 cell adhesion & 37 & 6.70 & $1.61 \mathrm{E}-8$ & $\begin{array}{l}\text { Ptprf, Fat2, Dscam, Perp, Fbln7, Cntnap2, Atp1b1, Cd24a, Ptk7, } \\
\text { Epha1, Fn1, Pkp1, Lamc2, Col7a1, Lama1, Col13a1, Grhl2, Nrxn3, } \\
\text { Itgb6, Fermt1, Spp1, Tenm2, Itgb4, Cd9, Celsr2, Cadm4, Cdh3, } \\
\text { Cdh11, Col16a1, Col8a1, Cdh1, Itga8, Ephb1, Spon1, Nectin4, } \\
\text { Flrt2, and Col14a1 }\end{array}$ \\
\hline GO:0042060 wound healing & 16 & 2.89 & 1.84E-8 & $\begin{array}{l}\text { Dsp, Arhgef19, Timp1, Tgfa, Msx2, Bnc1, Cdh3, Wnt5b, Tgfb3, } \\
\text { Pak1, Plau, Ptk7, Erbb2, Fn1, Epb41 l4b, and Tgfb2 }\end{array}$ \\
\hline GO:0008285 negative regulation of cell proliferation & 32 & 5.79 & $2.51 \mathrm{E}-8$ & $\begin{array}{l}\text { Ptprf, Timp2, Tfap2b, Irf6, Gata3, Sfrp1, Hspa1a, Sox9, Tfap2a, } \\
\text { Runx1, Bnipl, Bmp7, Tgfb2, Vdr, Sfrp4, Scin, Cd9, Lif, Fgfr2, } \\
\text { Msx2, Wnk2, Sfrp2, Frzb, Slit2, Tgfb3, Ptprz1, Plk5, Ovol2, Ror2, } \\
\text { Nos1, Rerg, and Sox4 }\end{array}$ \\
\hline $\begin{array}{l}\text { GO:0090090 negative regulation of canonical Wnt } \\
\text { signaling pathway }\end{array}$ & 14 & 2.53 & $2.33 \mathrm{E}-6$ & $\begin{array}{l}\text { Sox10, Nkd2, Cthrc1, Sfrp2, Wnt5b, Dkk3, Sfrp1, Frzb, Lrp4, Cdh1, } \\
\text { Sox9, Ror2, Wnt4, and Sfrp4 }\end{array}$ \\
\hline GO:0007275 multicellular organism development & 53 & 9.60 & $3.22 \mathrm{E}-6$ & $\begin{array}{l}\text { Shroom3, Sfrp1, Ngef, Lrp4, Enah, Tbx3, Dbn1, Sfrp4, Prrx2, } \\
\text { Ephb3, Plekhb1, Lmx1b, Celsr2, Dkk3, Frzb, Slit2, Wnt2, Ovol2, } \\
\text { Anpep, Wnt4, Ano1, Tmem100, Grem2, Elf3, Foxa1, Dact2, Fzd7, } \\
\text { Wnt5b, Irx4, Kdf1, Fzd10, Mdfi, Bmp7, Mycbpap, Sema3d, } \\
\text { Col13a1, Wnt7b, Vdr, Cited1, Smpd3, Msx2, Sfrp2, Itga8, Krt8, } \\
\text { Eya2, Irx3, Trp63, Alx4, Ror2, Cxcl17, Dmbt1, Flrt2, and Islr2 }\end{array}$ \\
\hline GO:0061180 mammary gland epithelium development & 6 & 1.08 & 4.66E-6 & Wnt2, Atp2c2, Prlr, Msx2, Wnt4, and Wnt7b \\
\hline GO:0008284 positive regulation of cell proliferation & 34 & 6.15 & $5.53 \mathrm{E}-6$ & $\begin{array}{l}\text { Tfap2b, Ccnd1, Ptn, Cxcr2, Sfrp1, Sox9, Tbx3, Pgr, Plau, Epcam, } \\
\text { Epha1, Erbb2, Fn1, Areg, Lamc2, Tgfb2, Akr1c18, Wnt7b, Gas1, } \\
\text { Lif, Tgfa, Timp1, Rab25, Fgfr2, Sfrp2, Wnt2, Id4, Pak1, Efemp1, } \\
\text { Folr2, Osr2, Cldn7, Klf5, Sox4 }\end{array}$ \\
\hline GO:0030855 epithelial cell differentiation & 11 & 1.99 & $8.26 \mathrm{E}-6$ & $\begin{array}{l}\text { Krt14, Muc1, Upk2, Trp63, Elf3, Aldoc, Vil1, Fgfr2, Bmp7, Bdh2, } \\
\text { and Ehf }\end{array}$ \\
\hline $\begin{array}{l}\text { GO:0045669 positive regulation of osteoblast } \\
\text { differentiation }\end{array}$ & 11 & 1.99 & $9.46 \mathrm{E}-6$ & $\begin{array}{l}\text { Id4, Cd276, Trp63, Cthrc1, Msx2, Bmp7, Sfrp2, Wnt4, Ltf, Wnt7b, } \\
\text { and Fbn2 }\end{array}$ \\
\hline
\end{tabular}



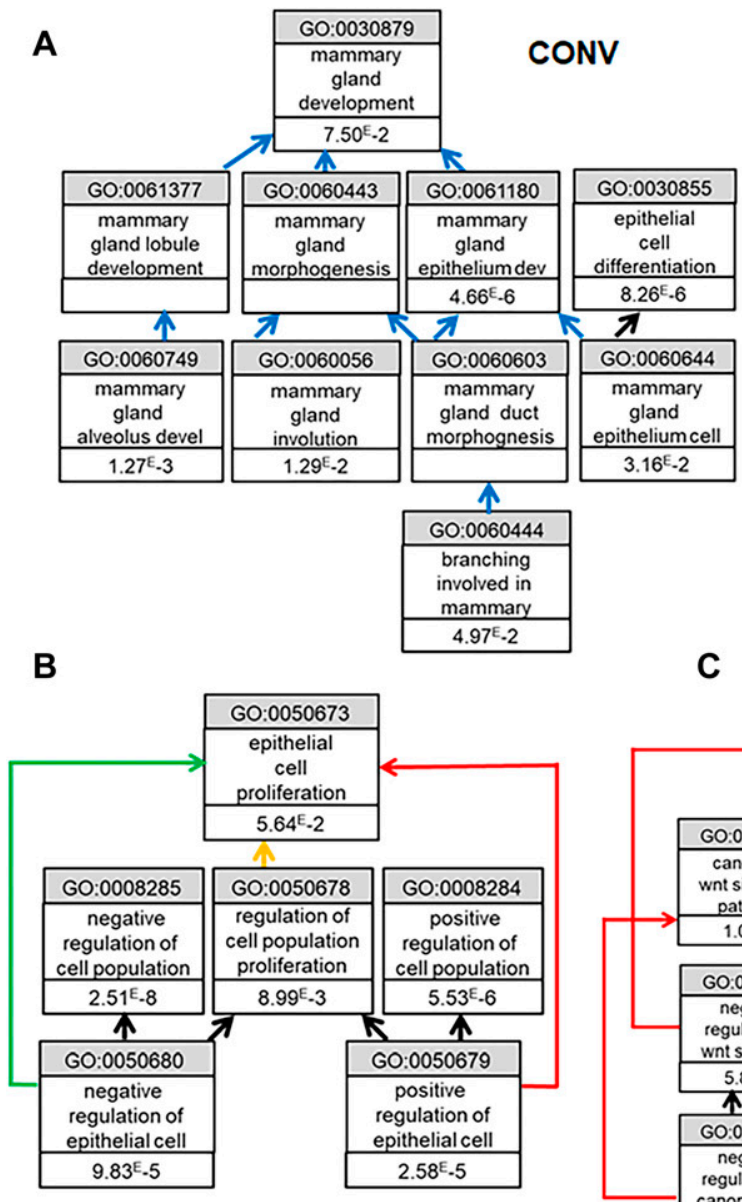

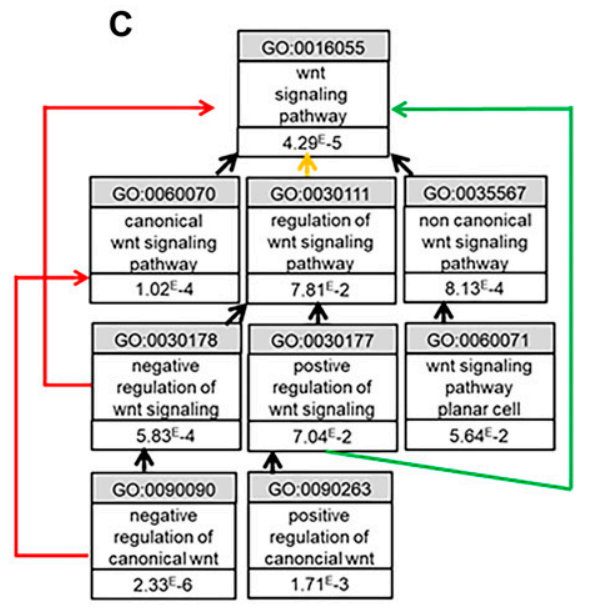

E

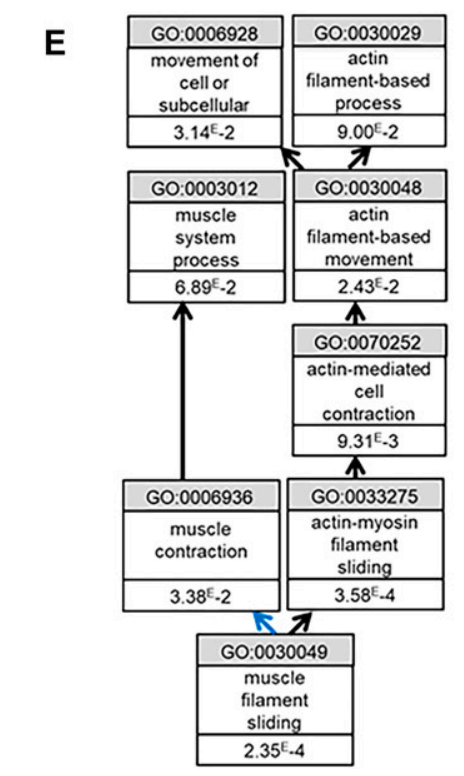

Figure 5. Cxcr2 KO affects mammary gland function in conventional conditions.

(A, B, C) Gene Ontology analysis of down-regulated genes in the mammary gland of KO animals. Black arrows mean "is a." Blue arrows mean is "part of." Green arrow means "positively regulates." Red arrow means "negatively regulates." Yellow arrow means "regulates." (A) Biological process related to mammary gland function. (B) Similar analysis as in (B) in terms of cell proliferation. (C) Similar analysis as in (B) in terms of Wht signaling. (D, E) Gene Ontology analysis of up-regulated genes in the mammary gland of KO animals. (D) Biological processes related to chemotaxis. (E) Biological processes related to muscle function.

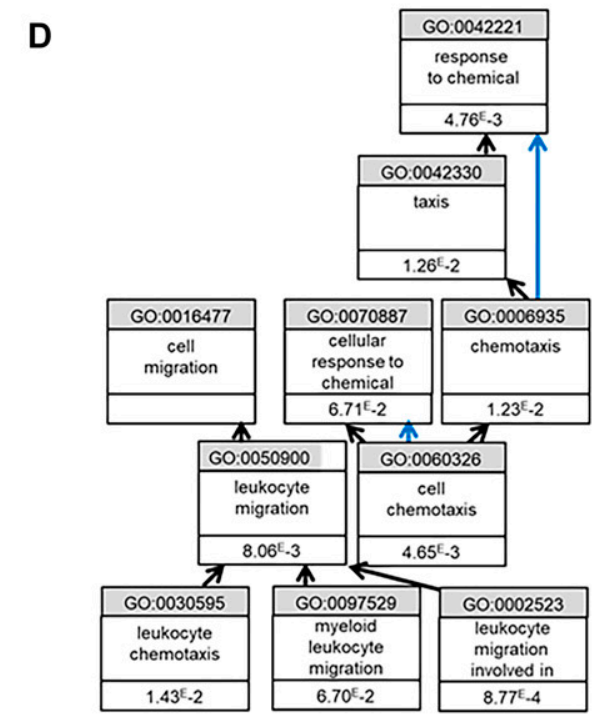

the uteri of Cxcr2 KO animals housed conventionally, with global reductions in uterine size, and decreases in the endometrial thickness and the number of glands present, suggesting uterus in arrest not cycling under hormonal stimulation.
The mammary glands of Cxcr2 KO females housed in conventional facilities were also minimally developed, harboring a phenotype close to that of juvenile animals with a rudimentary duct branching. In contrast, in SOPF conditions, mammary gland development appeared 
Table 4. Most enriched pathways for genes up-regulated in the mammary gland of KO versus WT conventional animals.

\begin{tabular}{|c|c|c|c|c|}
\hline GO biological process term & Count & $\%$ & $P$-value & Genes \\
\hline GO:0030049 muscle filament sliding & 3 & 5.55 & $2.35 \mathrm{E}-4$ & Myh6, Myh7, and Tnnc1 \\
\hline $\begin{array}{l}\text { GO:0055010 ventricular cardiac muscle tissue } \\
\text { morphogenesis }\end{array}$ & 4 & 7.40 & $3.52 \mathrm{E}-4$ & Myl3, Myh6, Myh7, and Tnnc1 \\
\hline GO:0033275 actin-myosin filament sliding & 3 & 5.55 & $3.58 \mathrm{E}-4$ & Myh6, Myh7, and Tnnc1 \\
\hline $\begin{array}{l}\text { GO:0003229 ventricular cardiac muscle tissue } \\
\text { development }\end{array}$ & 4 & 7.40 & 5.07E-4 & Myl3, Myh6, Myh7, and Tnnc1 \\
\hline GO:0055008 cardiac muscle tissue morphogenesis & 4 & 7.40 & 7.97E-4 & Myl3, Myh6, Myh7, and Tnnc1 \\
\hline $\begin{array}{l}\text { GO:0002523 leukocyte migration involved in inflammatory } \\
\text { response }\end{array}$ & 3 & 5.55 & $8.77 \mathrm{E}-4$ & S100a9, Ffar2, and S100a8 \\
\hline
\end{tabular}

similar to that of WT animals. Interestingly, even in aged animals, no further development of the mammary gland could be observed in Cxcr2 KO females housed in conventional facilities (data not shown). At the molecular level, the mammary gland of Cxcr2 KO animals showed a clear down-regulation of the expression of genes involved in mammary gland development and differentiation, epithelial cell proliferation, wound healing, and Wnt signaling (including lactoferrin, Gata3, cyclin D1, Erbb2, Elf5, Epcam, Prlr, Krt15, Epcam, Wnt4, and Wnt5b). For a number of the down-regulated genes, invalidation studies in mice have shown that these genes (Prlr, cyclin D1, Erbb2, and Elf5) are crucial for mammary gland development (Bole-Feysot et al, 1998; Zhou et al, 2005; Howlin et al, 2006). The Wnt pathway plays a pivotal role in orchestrating proper mammary gland development and maintenance (Jarde \& Dale, 2012), and this pathway was impaired in Cxcr2 KO animals. The Wnt pathway is a major paracrine mediator of hormonal action, through Wnt4 and Areg pathways in particular (Brisken et al, 2000; Ciarloni et al, 2007), both of which are down-regulated in the mammary glands of Cxcr2 KO animals housed conventionally. The general scheme of Wnt action in the mammary gland is based on the activation of estrogen receptor alpha (ER $\alpha)$ - positive luminal cells, which in turn are stimulated by steroid hormones to release Wnt ligands that will act directly or indirectly on the myoepithelial compartment (Jarde \& Dale, 2012).

The collection of evidence gathered herein, for possible hormonal perturbations affecting Cxcr2 KO animals housed in conventional conditions, including mammary, ovary, and uterus histologic changes and molecular defects, led us to evaluate steroid hormone levels in these animals. We observed, in particular, a decrease in serum progesterone levels, which could reflect the absence of corpora lutea in the ovary of these animals, the main site of production of progesterone.

To demonstrate the role of the hormonal environment in the phenotype observed for $\mathrm{CxC} 2 \mathrm{KO}$ animals in conventional conditions, we decided to perform ovarian transplantation experiments. By transplanting the ovary of $\mathrm{CxCr} 2 \mathrm{KO}$ animals into the ovarian bursa of ovariectomized WT animals, we observed that KO ovaries could display a normal histology, close to WT ovaries, with the presence of corpora lutea, a sign of successive ovulations. Moreover, the transplanted animals had their fertility restored and were able to give birth to viable mice. Similarly, transplantation of the mammary glands of $\mathrm{Cxcr} 2 \mathrm{KO}$ animals into a WT context also led to the development of normal gland development with correct ductal branching. Taken together, this confirms that the hormonal context of WT animals is sufficient to restore a correct function and development of the Cxcr2 KO ovary and mammary gland. As a function of the ovary, the uterus and the mammary gland are tightly controlled by steroid hormones, and at a higher level, by pituitary hormones, we measured the circulating levels of FSH, LH, GH, and PRL. The levels of these four pituitary hormones were markedly decreased in $\mathrm{CxCr} 2 \mathrm{KO}$ animals housed in conventional conditions compared with WT animals, suggesting an alteration to pituitary function. Treating the KO animals with FSH and LH to restore fertility could be interesting issue, but it is likely that the timing will be critical and difficult to assess because of the lack of cycling of these animals.

Transcriptomic and GO analysis of the pituitary of WT and Cxcr2 $\mathrm{KO}$ animals revealed as expected, a down-regulation of genes involved in the control of circadian rhythm, ovulation control, and gonad development; which could account for dysregulation of pituitary hormones. This includes genes such as Esr1 (estrogen receptor alpha), Pgr (progesterone receptor), RMB4 (required for the translational activation of PER1 mRNA in response to circadian clock) (Markus \& Morris, 2009), Crebbp (CREB-Binding Protein, involved in circadian clock) (Rexach et al, 2012), Foxo3 (a regulator of circadian clock) (Chaves et al, 2014). Moreover, one could expect behavior alterations of Cxcr2 $\mathrm{KO}$ animals based on the downregulation of a number of genes controlling behavior, including for instance Crhbp (corticotropin-releasing hormone binding protein) (Ketchesin et al, 2017), Oprk1 (opioid receptor, kappa 1) (Loh et al, 2017), or Vip (vasoactive intestinal polypeptide) (Hill, 2007). The dysfunction of the Cxcr2 KO pituitary involves presumably defects in synapse function, as well as ion transport. Indeed, we report a down-regulation of a number of genes involved in calcium, sodium, and potassium transport or in synaptic transmission such as Vip, Oprk1, Kcnb2 (potassium voltage gated channel, Shab-related subfamily, member 2) or Trpc6 (transient receptor potential cation channel, subfamily C, member 6), Cacna1g (calcium channel, voltage-dependent, $T$ type, alpha $1 \mathrm{G}$ subunit). Synaptic alteration includes anterograde trans-synaptic signaling, long-term synaptic potentiation, and synaptic plasticity and could have major effects on neuronal connections. In addition to down-regulation of the pathways mentioned above, other pathways appeared up-regulated in the pituitary of $\mathrm{CxCr} 2 \mathrm{KO}$ animals. This includes in particular aggregation and adhesion of leukocytes as well as up-regulation of 

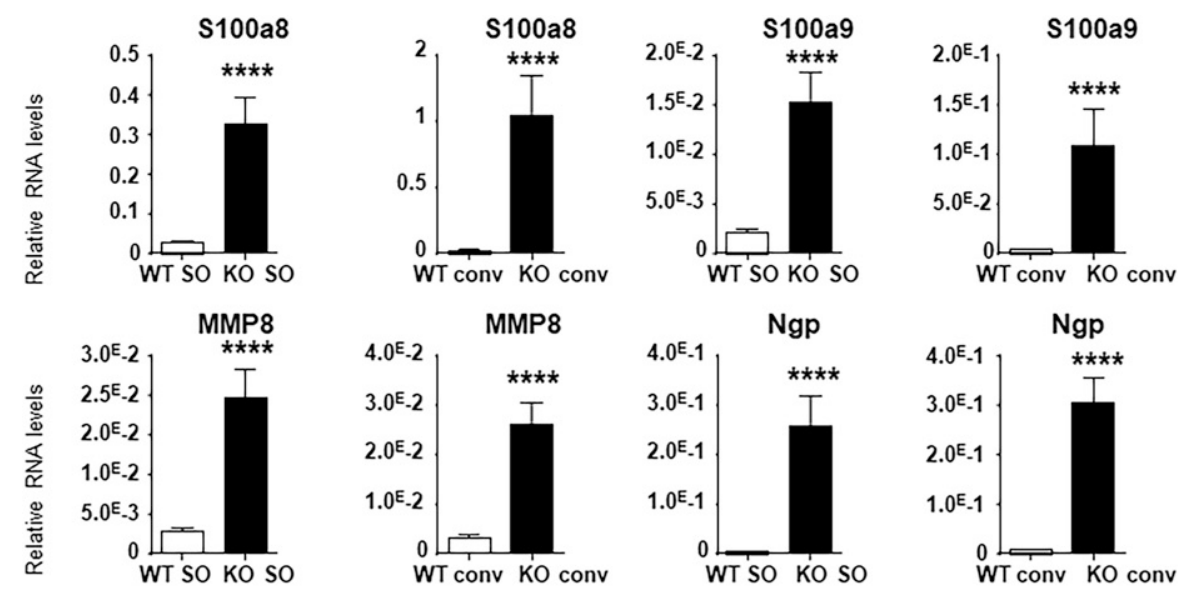

Figure 6. Cxcr2 KO affects mammary gland transcriptome in conventional conditions.

Measure of RNA levels by real-time PCR of a set of genes in the mammary gland of WT and KO animals in conventional or SOPF conditions. Results represent the mean the mean \pm SEM of at least 12 animals (Mann-Whitney test, NS, nonsignificant, ${ }^{*} P<0.05$, ${ }^{* *} P<$ $\left.0.01,{ }^{* * *} P<0.001,{ }^{* * * *} P<0.0001\right)$.
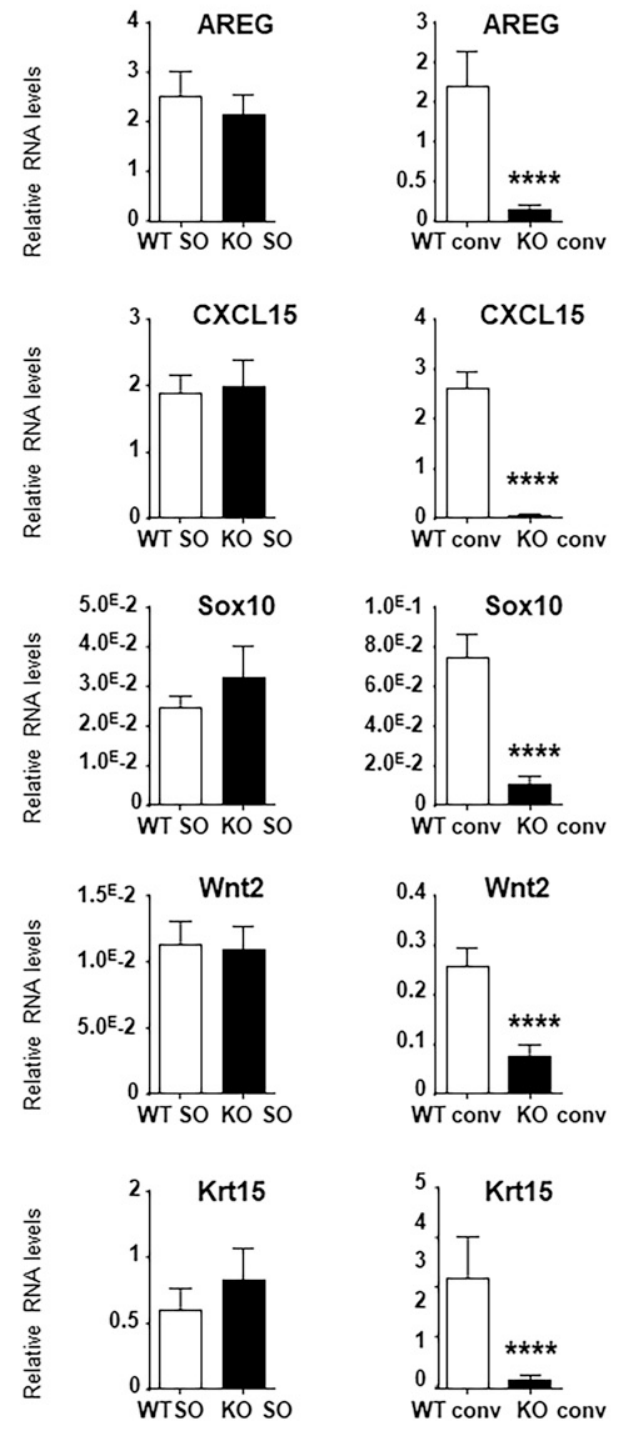
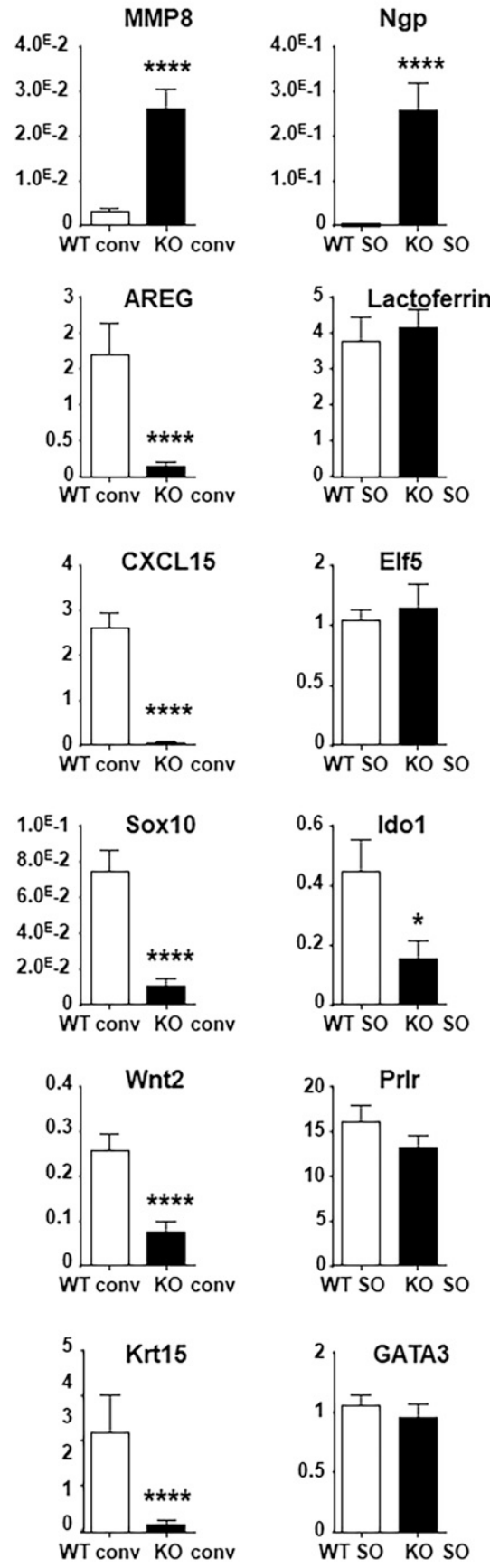

chemotaxis, extravasation, tethering, or rolling and also leukocyte activation, proliferation, and differentiation. When comparing our RNAseq results with signatures for B lymphocytes, T lymphocytes, macrophages, and neutrophils from Nirmal et al (2018), it appears
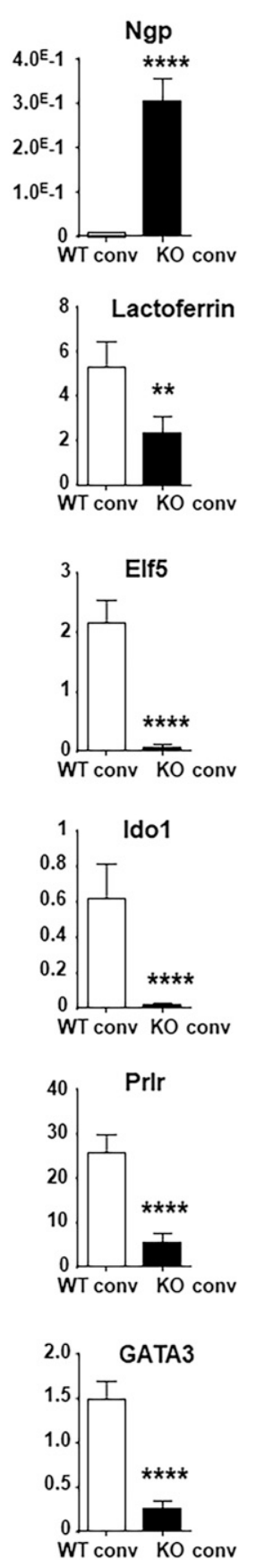

that there is a T lymphocyte enrichment (21\%) and to a lesser extent of B lymphocytes (13.5\%), macrophages (12.8\%), and neutrophils $(6.4 \%)$ (Table S1). This possible infiltration of T and B lymphocytes in the pituitary could be reminiscent of autoimmune hypophysitis 
A
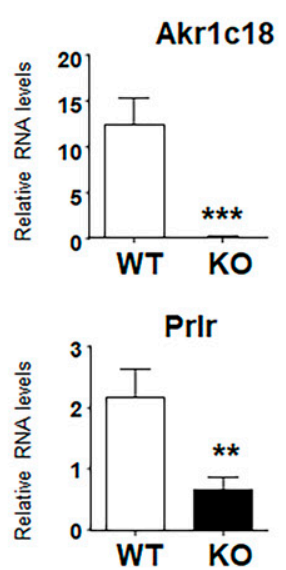

CONV
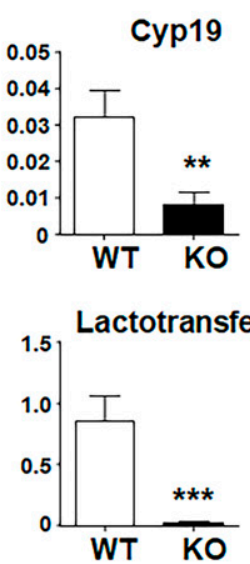

D

WT mouse

CONV

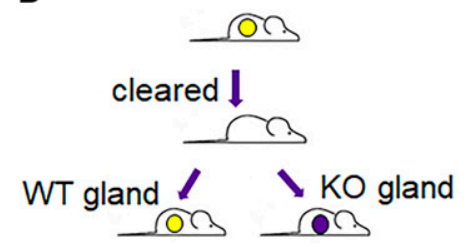

No transplant

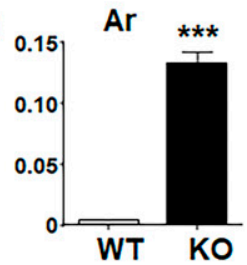

WT transplant
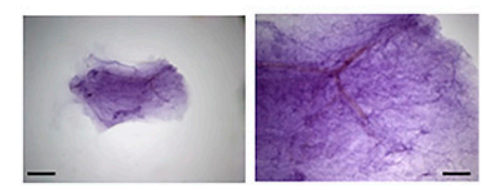

$\mathrm{KO}$ transplant
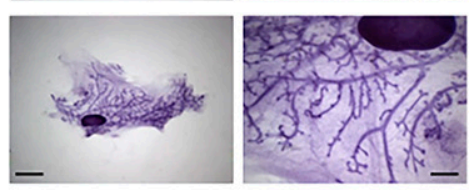

B

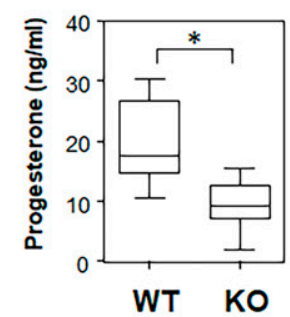

CONV
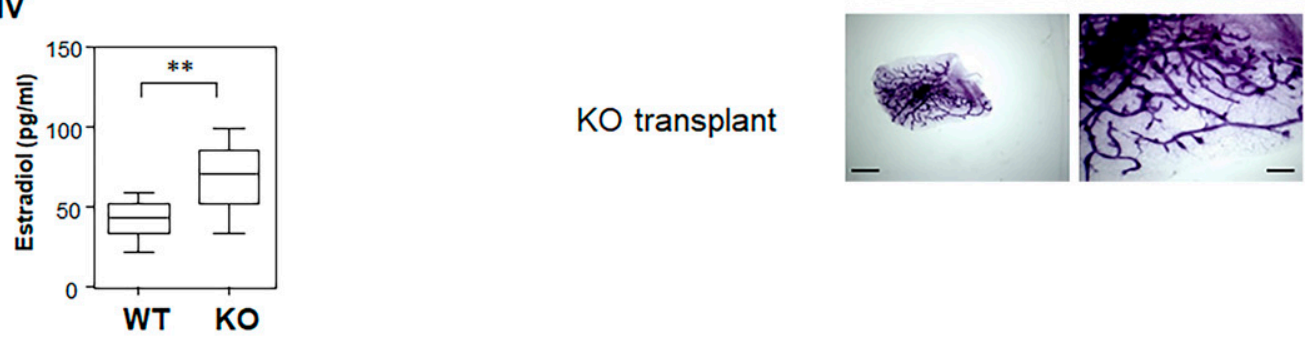

C

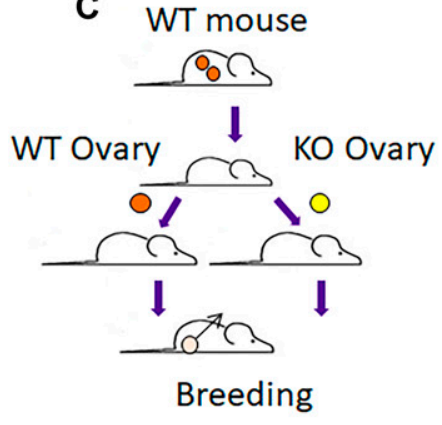

\section{CONV}

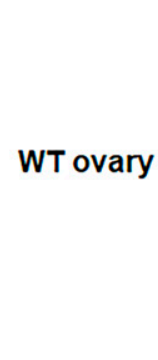

KO ovary

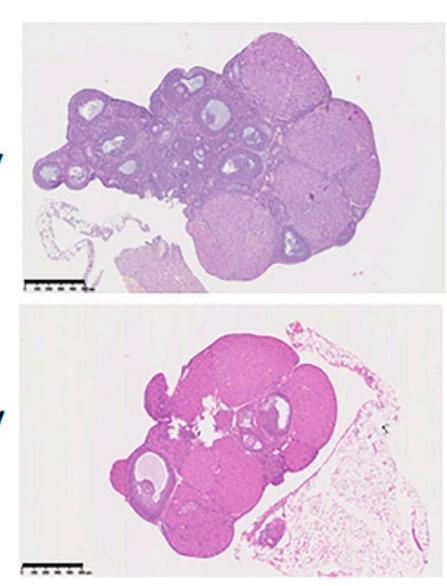

$\%$ of successful breeding

\begin{tabular}{|l|c|}
\hline WT donor & $100 \%$ \\
\hline KO donor & $81 \%$ \\
\hline
\end{tabular}

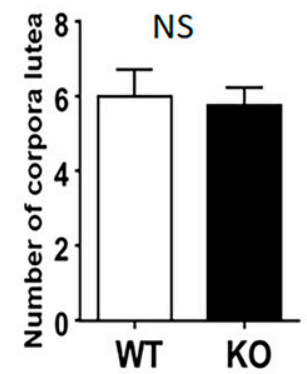

Figure 7. Cxcr2 KO display an alteration of hormonal function in conventional conditions.

(A) Measure of RNA levels of a set of genes in the ovary of WT and KO animals in conventional conditions by real-time PCR. Results represent the mean the mean \pm SEM of at least seven animals (Mann-Whitney test, NS, nonsignificant, ${ }^{*} P<0.05$, ${ }^{* *} P<0.01$, ${ }^{* * *} P<0.001$ ). (B) Serum levels of estradiol and progesterone in WT and KO mice in conventional conditions. Box and whiskers represent the min and max of at least 10 animals (Mann-Whitney test, NS, nonsignificant, ${ }^{*} P<0.05$, ${ }^{\star \star} P<0.01$ ). (C) Left panel: strategy of ovary transplantation. A Cxcr2 WT mouse was ovariectomized and reimplanted with either WT or KO ovary from a conventional facility. Once the graft was established, females were bred with WT males to evaluate their fertility. Right panel: Histology of the transplanted CXcr2 WT and Cxcr2 KO ovaries. Representative images of hematoxylin-eosin-stained ovaries at a 5x magnification are shown here. Scale bars: $500 \mu \mathrm{m}$. The \% of successful breeding of transplanted recipient females is indicated. Fisher's exact test shows no difference between WT and KO successful breeding $(P=0.5147)$. The number of corpora lutea in WT or KO transplanted ovaries is also presented and shows no statistical difference (Mann-Whitney test, NS). (D) Left panel: strategy of mammary gland transplantation. The mammary gland fat pads of Cxcr2 WT mice were cleared and transplanted with either Cxcr2 WT or Cxcr2 KO mammary gland. Right panel: Whole mounts of mammary glands of recipient mice after no transplantation or transplantation with WT or KO mammary glands. Scale bars: $5 \mathrm{~mm}$ (left panel) or $1.3 \mathrm{~mm}$ (right panel). 


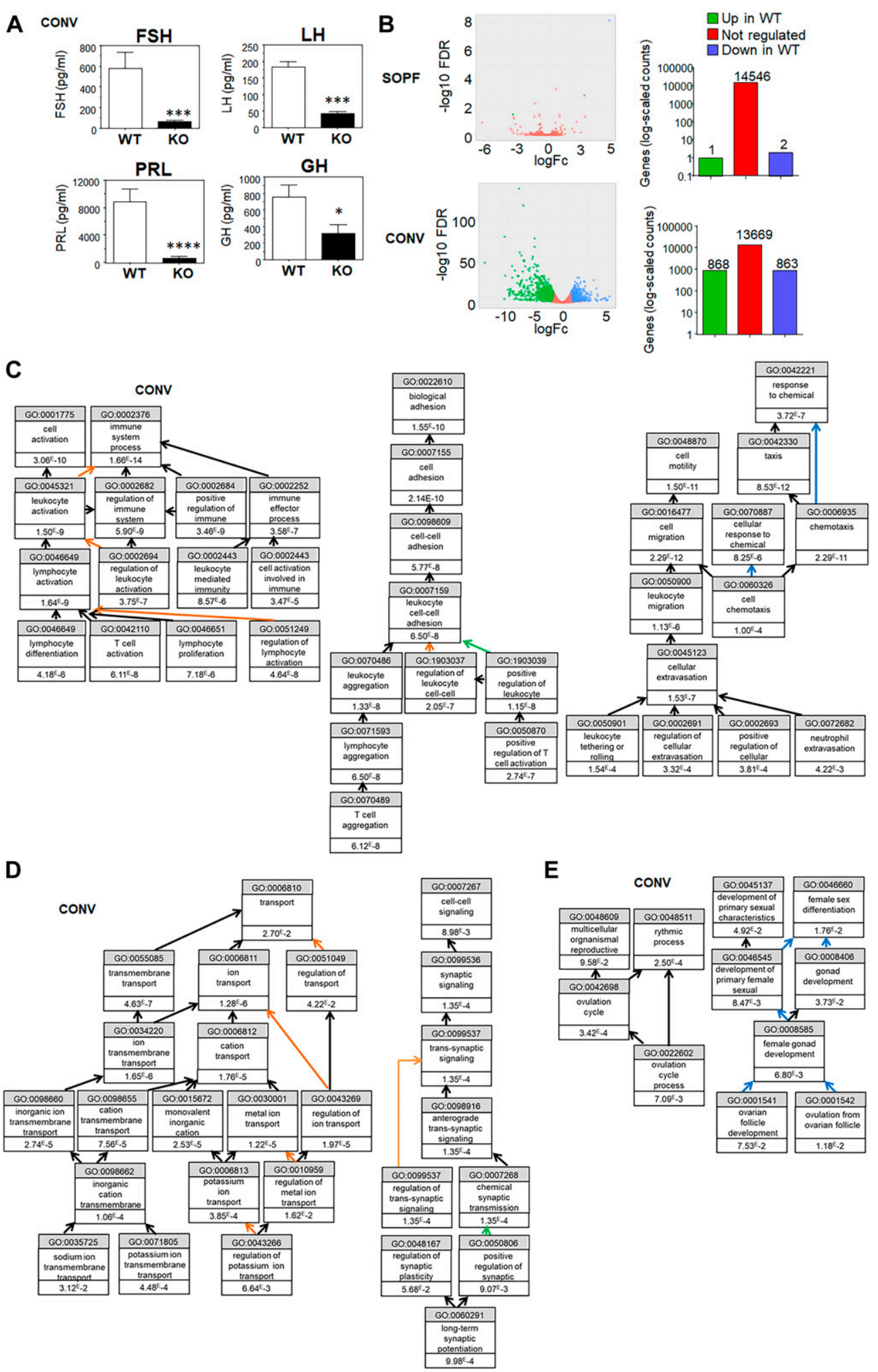

Figure 8. Circulating pituitary hormones and transcriptome in the pituitary of KO animals are drastically affected in conventional housing conditions.

(A) Serum levels of pituitary hormones FSH, LH, PRL, and GH. Results represent the mean \pm SEM of at least 14 animals

(Mann-Whitney test, NS, nonsignificant, ${ }^{*} P<0.05,{ }^{* *} P<0.01,{ }^{* \star *} P<0.001,{ }^{* \star * \star} P<$ 0.0001). (B) Left panel: the volcano plots show the global changes in RNA expression patterns for WT versus KO pituitary in SOPF or conventional conditions. Data represent analysis of cpm estimates with a log of fold change of more than 1.5fold and $P<0.05$ of 3 animals per group. Right panel: Number of differentially regulated genes for the same analysis. (C) Gene Ontology (GO) analysis of upregulated genes in the pituitary of KO animals in conventional conditions. (D) GO analysis of down-regulated genes in the pituitary of $\mathrm{KO}$ animals in conventional conditions linked to synapse function and ion transport. (E) GO analysis of downregulated genes in the pituitary of $\mathrm{Cxcr2}$ $\mathrm{KO}$ animals in conventional conditions linked to reproduction.

D

$(\mathrm{AH})$, which is an inflammatory disease of the pituitary gland, and particularly in relation to lymphocytic hypophysitis (Bellastella et al, 2016). AH can lead to atrophy of the pituitary and to hypopituitrism, which is in agreement with what we observed for $\mathrm{Cxcr} 2$
$\mathrm{KO}$ animals in conventional conditions. Functional disturbances observed in humans with $\mathrm{AH}$ include headaches, visual disturbances, hypothyroidism, hypogonadism, and an inability to lactate (Caturegli et al, 2005). 
Table 5. Most enriched pathways for genes up-regulated in the pituitary of KO versus WT conventional animals.

\begin{tabular}{|c|c|c|c|c|}
\hline GO biological process term & Count & $\%$ & $P$-value & Genes \\
\hline $\begin{array}{l}\text { GO:0044707 single-multicellular } \\
\text { organism process }\end{array}$ & 384 & 45.33 & $5.40 E-29$ & 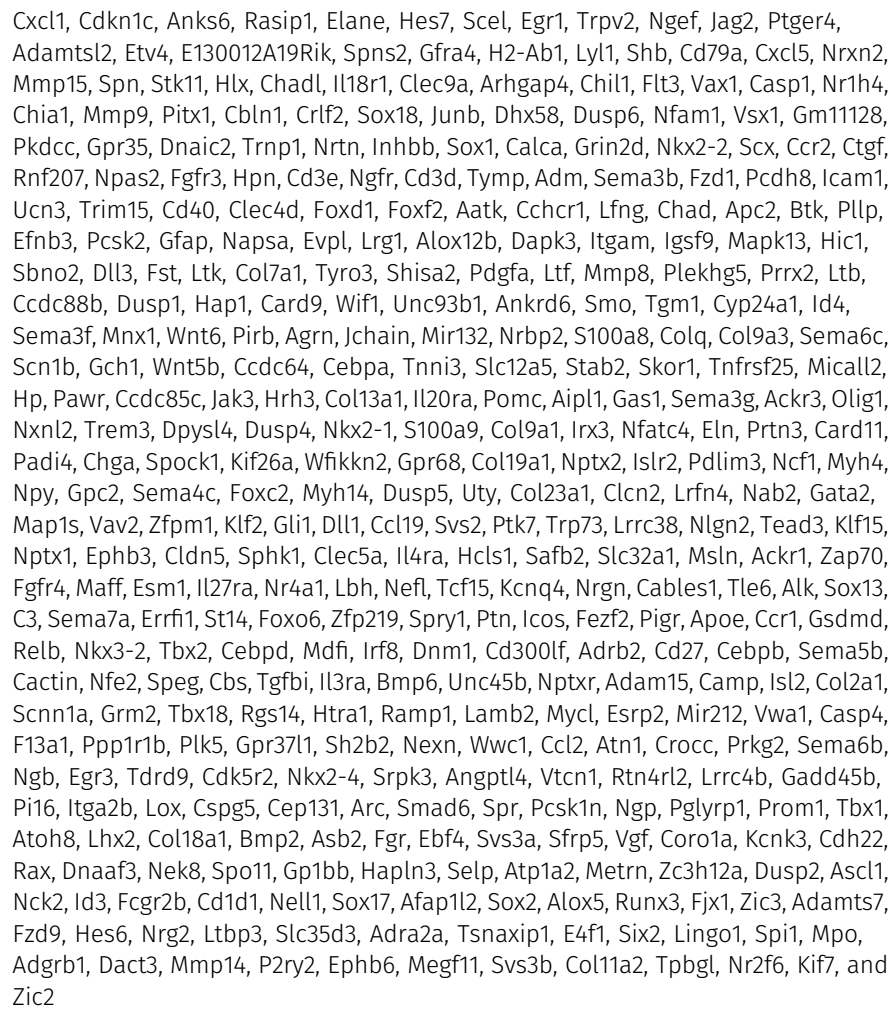 \\
\hline $\begin{array}{l}\text { GO:0007275 multicellular organism } \\
\text { development }\end{array}$ & 320 & 37.78 & $2.52 \mathrm{E}-21$ & $\begin{array}{l}\text { Cxcl1, Cdkn1c, Anks6, Rasip1, Hes7, Scel, Egr1, Trpv2, Ngef, Jag2, Ptger4, Adamtsl2, } \\
\text { Etv4, E130012A19Rik, Spns2, H2-Ab1, Lyl1, Shb, Cd79a, Cxcl5, Nrxn2, Mmp15, Spn, Stk11, } \\
\text { Hlx, Chadl, Il18r1, Arhgap4, Chil1, Flt3, Vax1, Nr1h4, Mmp9, Pitx1, Cbln1, Sox18, Junb, } \\
\text { Dusp6, Nfam1, Vsx1, Gm11128, Pkdcc, Dnaic2, Trnp1, Nrtn, Inhbb, Sox1, Calca, Nkx2-2, } \\
\text { Scx, Ccr2, Ctgf, Fgfr3, Hpn, Cd3e, Ngfr, Cd3d, Tymp, Adm, Sema3b, Fzd1, Pcdh8, Icam1, } \\
\text { Clec4d, Cd40, Foxd1, Foxf2, Aatk, Cchcr1, Lfng, Chad, Apc2, Btk, Pllp, Efnb3, Pcsk2, } \\
\text { Gfap, Evpl, Lrg1, Alox12b, Dapk3, Itgam, Igsf9, Hic1, Sbno2, Dll3, Fst, Ltk, Col7a1, Tyro3, } \\
\text { Pdgfa, Shisa2, Ltf, Mmp8, Prrx2, Ltb, Hap1, Dusp1, Wif1, Ankrd6, Smo, Tgm1, Id4, } \\
\text { Sema3f, Mnx1, Wnt6, Pirb, Agrn, Mir132, Nrbp2, S100a8, Colq, Col9a3, Sema6c, Scn1b, } \\
\text { Wnt5b, Ccdc64, Cebpa, Tnni3, Slc12a5, Skor1, Tnfrsf25, Micall2, Hp, Pawr, Ccdc85c, } \\
\text { Jak3, Col13a1, Gas1, Sema3g, Ackr3, Olig1, Dpysl4, Dusp4, Nkx2-1, S100a9, Col9a1, Irx3, } \\
\text { Nfatc4, Eln, Prtn3, Card11, Spock1, Kif26a, Wfikkn2, Gpr68, Col19a1, Islr2, Pdlim3, } \\
\text { Myh4, Npy, Gpc2, Sema4c, Foxc2, Myh14, Dusp5, Uty, Clcn2, Lrfn4, Nab2, Gata2, Map1s, } \\
\text { Vav2, Zfpm1, Klf2, Gli1, Dll1, Ccl19, Ptk7, Trp73, Lrrc38, Nlgn2, Tead3, Klf15, Nptx1, } \\
\text { Ephb3, Cldn5, Sphk1, Clec5a, Il4ra, Hcls1, Slc32a1, Safb2, Msln, Zap70, Fgfr4, Maff, } \\
\text { Esm1, Il27ra, Nr4a1, Lbh, Nefl, Tcf15, Kcnq4, Nrgn, Cables1, Tle6, Sox13, Alk, C3, } \\
\text { Sema7a, Errfi1, St14, Foxo6, Zfp219, Spry1, Icos, Ptn, Fezf2, Apoe, Ccr1, Relb, Tbx2, } \\
\text { Nkx3-2, Cebpd, Mdfi, Irf8, Cd30olf, Adrb2, Cebpb, Cd27, Sema5b, Cactin, Nfe2, Speg, } \\
\text { Tgfbi, Cbs, Il3ra, Bmp6, Unc45b, Nptxr, Camp, Adam15, Isl2, Col2a1, Tbx18, Rgs14, } \\
\text { Htra1, Ramp1, Mycl, Esrp2, Lamb2, Mir212, Casp4, Plk5, Sh2b2, Nexn, Gpr37l1, Ccl2, } \\
\text { Atn1, Sema6b, Ngb, Egr3, Tdrd9, Cdk5r2, Nkx2-4, Srpk3, Angptl4, Rtn4rl2, Lrrc4b, } \\
\text { Gadd45b, Pi16, Lox, Arc, Cep131, Cspg5, Smad6, Spr, Prom1, Ngp, Pglyrp1, Tbx1, Atoh8, } \\
\text { Lhx2, Col18a1, Bmp2, Asb2, Ebf4, Sfrp5, Vgf, Kcnk3, Cdh22, Rax, Dnaaf3, Nek8, Spo11, } \\
\text { Hapln3, Metrn, Zc3h12a, Dusp2, Ascl1, Nck2, Id3, Cd1d1, Nell1, Sox17, Sox2, Runx3, Fjx1, } \\
\text { Zic3, Adamts7, Fzd9, Nrg2, Hes6, Ltbp3, Tsnaxip1, E4f1, Six2, Lingo1, Spi1, Adgrb1, } \\
\text { Dact3, Mmp14, P2ry2, Megf11, Col11a2, Tpbgl, Nr2f6, Kif7, and Zic2 }\end{array}$ \\
\hline
\end{tabular}

(Continued on following page) 


\begin{tabular}{|c|c|c|c|c|}
\hline $\begin{array}{l}\text { GO:0048856 anatomical structure } \\
\text { development }\end{array}$ & 340 & 40.14 & 2.177E-19 & 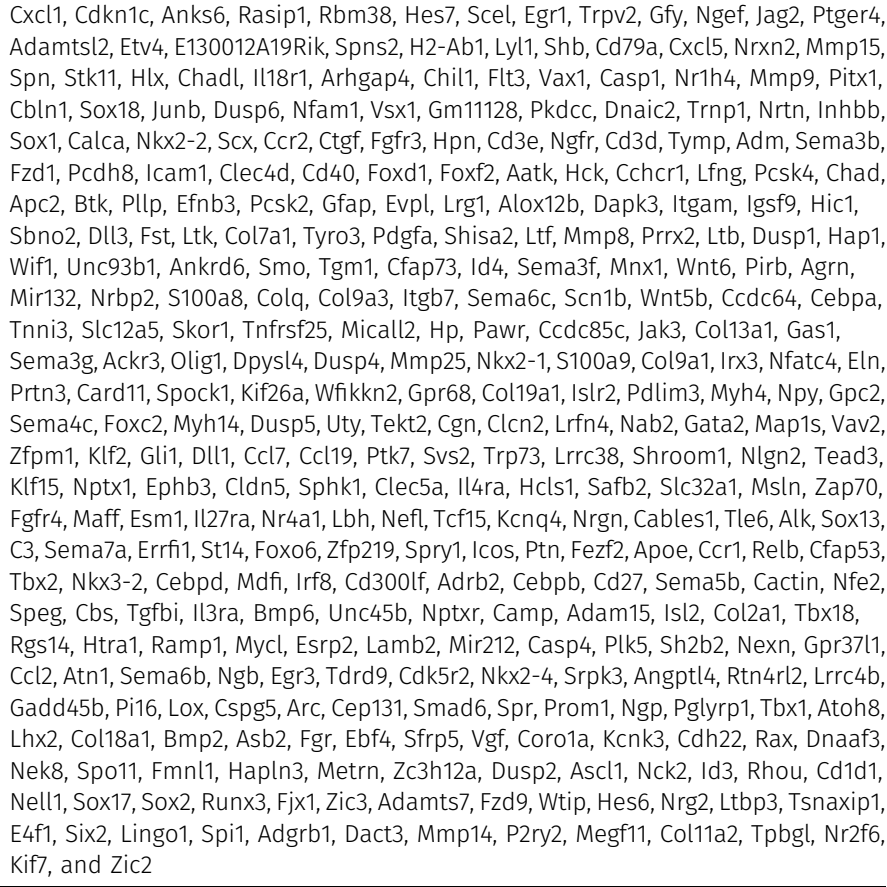 \\
\hline $\begin{array}{l}\text { GO:0051239 regulation of multicellular } \\
\text { organismal process }\end{array}$ & 202 & 23.84 & $2.79 \mathrm{E}-19$ & $\begin{array}{l}\text { Cxcl1, Cdkn1c, Elane, Hes7, Egr1, Trpv2, Ngef, Ptger4, Etv4, Gfra4, Shb, Cxcl5, Spn, } \\
\text { Stk11, Hlx, Chadl, Il18r1, Clec9a, Flt3, Arhgap4, Chil1, Vax1, Casp1, Nr1h4, Chia1, Cbln1, } \\
\text { Mmp9, Dusp6, Dhx58, Nfam1, Pkdcc, Gpr35, Inhbb, Calca, Grin2d, Nkx2-2, Ccr2, Scx, } \\
\text { Ctgf, Rnf207, Fgfr3, Hpn, Cd3e, Ngfr, Adm, Sema3b, Fzd1, Icam1, Trim15, Foxd1, Cd40, } \\
\text { Aatk, Lfng, Chad, Btk, Gfap, Lrg1, Alox12b, Mapk13, Fst, Dll3, Ltk, Pdgfa, Ltf, Ltb, } \\
\text { Ccdc88b, Hap1, Card9, Unc93b1, Ankrd6, Smo, Id4, Sema3f, Wnt6, Agrn, Mir132, Colq, } \\
\text { Sema6c, Scn1b, Cebpa, Tnni3, Stab2, Pawr, Jak3, Il20ra, Pomc, Sema3g, S100a9, Nkx2- } \\
\text { 1, Irx3, Nfatc4, Card11, Spock1, Chga, Gpr68, Islr2, Ncf1, Sema4c, Foxc2, Gata2, Vav2, } \\
\text { Gli1, Klf2, Zfpm1, Ccl19, Dll1, Ptk7, Trp73, Nlgn2, Tead3, Ephb3, Cldn5, Il4ra, Clec5a, } \\
\text { Sphk1, Hcls1, Ackr1, Fgfr4, Zap70, Maff, Il27ra, Lbh, Nefl, Tle6, Sox13, C3, Sema7a, Errfi1, } \\
\text { Foxo6, Spry1, Zfp219, Ptn, Fezf2, Ccr1, Apoe, Gsdmd, Relb, Tbx2, Nkx3-2, Cebpd, Irf8, } \\
\text { Cebpb, Cd27, Adrb2, Sema5b, Nfe2, Cactin, Bmp6, Camp, Isl2, Tbx18, Rgs14, Mycl, } \\
\text { Mir212, Casp4, Plk5, Gpr3711, Wwc1, Ccl2, Sema6b, Egr3, Snta1, Vtcn1, Lrrc4b, Pi16, Spr, } \\
\text { Smad6, Prom1, Pglyrp1, Ngp, Tbx1, Atoh8, Bmp2, Fgr, Sfrp5, Selp, Atp1a2, Metrn, } \\
\text { Zc3h12a, Ascl1, Fcgr2b, Id3, Afap112, Sox17, Nell1, Cd1d1, Sox2, Alox5, Adamts7, Fzd9, } \\
\text { Ltbp3, Adra2a, E4f1, Six2, Ling01, Spi1, Adgrb1, Dact3, Mmp14, P2ry2, Tpbgl, and Zic2 }\end{array}$ \\
\hline $\begin{array}{l}\text { GO:0007166 cell surface receptor } \\
\text { signaling pathway }\end{array}$ & 175 & 20.66 & 3.05E-19 & $\begin{array}{l}\text { Cxcl1, Cdkn1c, Gpc2, Sema4c, Foxc2, Uty, Hes7, Dlk2, Egr1, Gata2, Ngef, Gli1, Jag2, Ccl19, } \\
\text { Ccl7, Dll1, Ptk7, Adamtsl2, Grik5, Gfra4, Nlgn2, Shb, Cxcl5, Cd79a, Cd39, Spn, Ephb3, } \\
\text { Stk11, Cldn5, Il18r1, Lat2, Sphk1, Flt3, Ackr1, Lat, Zap70, Fgfr4, Esm1, Nr1h4, Rhbdf2, } \\
\text { Mmp9, Alk, Sema7a, Errfi1, Nfam1, Spry1, Lcn2, Gpr35, Nrtn, Inhbb, Pigr, Ccr1, Styk1, } \\
\text { Grin2d, Tbx2, Blk, Nkx2-2, Adgrg5, Fpr2, Ccr2, Scx, Ctgf, Mdfi, Fgfr3, Adrb2, Cd27, } \\
\text { Sema5b, Cactin, Il3ra, Cd3e, Bmp6, Adam15, Ngfr, Cd3d, Sema3b, Col2a1, Fzd1, Tbx18, } \\
\text { Rgs14, Icam1, Tle2, Foxd1, Cd40, Clec4d, Htra1, Hck, Matk, Gpr37l1, Sh2b2, Lfng, Chad, } \\
\text { Apc2, Ccl2, Btk, Efnb3, Sema6b, Lrg1, Pmaip1, Dapk3, Rtn4rl2, Itgam, Lrrc4b, Itgax, } \\
\text { Myo1g, Hic1, Itga2b, Ltbp4, Fst, Dll3, Arc, Ltk, Smad6, Shisa2, Pdgfa, Atoh8, Ltf, Ltb, } \\
\text { Prrx2, Bmp2, Hap1, Fgr, Frat2, Epha10, Wif1, Ankrd6, Smo, Sfrp5, Tspan33, Coro1a, } \\
\text { Igfbp4, Sema3f, Wnt6, Pirb, Podnl1, Ccl6, Ascl1, Nck2, Fcgr2b, Itgb7, Osmr, Afap1l2, } \\
\text { Sox17, Sema6c, Pear1, Sox2, Wnt5b, Runx3, Cebpa, Skor1, Fzd9, Rhbdf1, Hp, Nrg2, } \\
\text { Pawr, Ltbp3, Jak3, Adra2a, Il20ra, Mib2, Adgrb1, Gas1, Dact3, Sema3g, Mmp14, Ackr3, } \\
\text { Csf2rb, Ephb6, Nkx2-1, Tpbgl, Nfatc4, Kif7, Card11, Pdzd3, Wfikkn2, and Zic2 }\end{array}$ \\
\hline
\end{tabular}




\begin{tabular}{|c|c|c|c|c|}
\hline GO:0032502 developmental process & 345 & 40.73 & $1.51 \mathrm{E}-18$ & 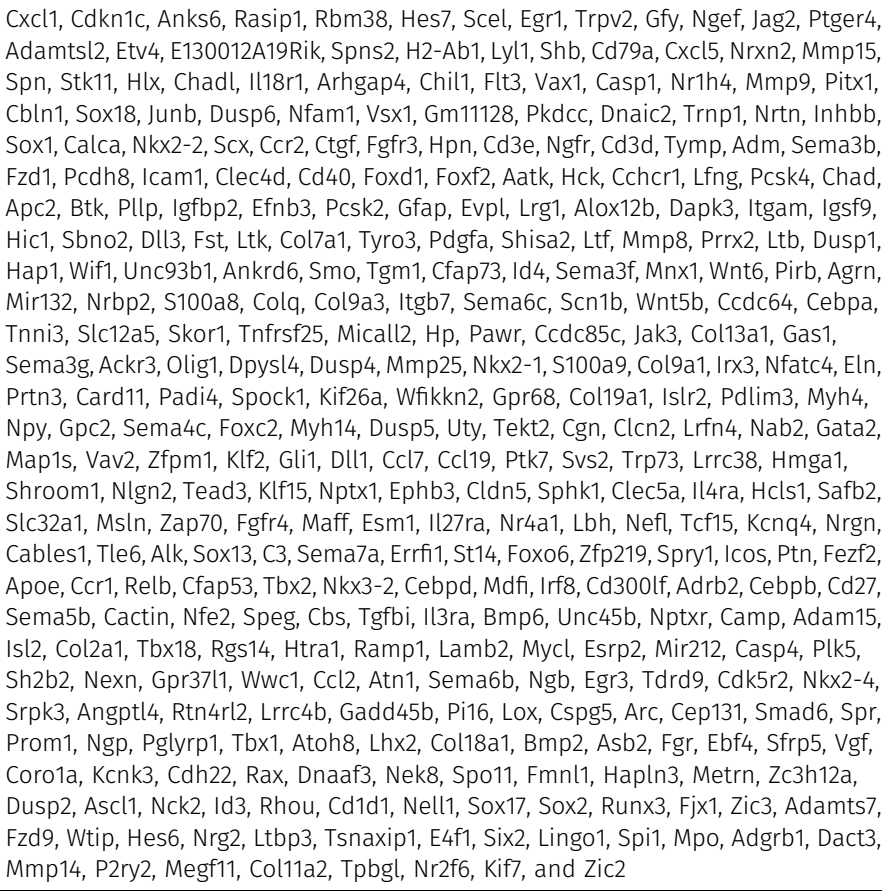 \\
\hline $\begin{array}{l}\text { GO:0051240 positive regulation of } \\
\text { multicellular organismal process }\end{array}$ & 136 & 16.05 & $2.07 E-18$ & $\begin{array}{l}\text { Cxcl1, Elane, Foxc2, Egn1, Trpv2, Gata2, Zfpm1, Gli1, Dll1, Ccl19, Ptger4, Ptk7, Trp73, } \\
\text { Nlgn2, Tead3, Shb, Cxcl5, Spn, Ephb3, Stk11, Hlx, Il18r1, Il4ra, Clec5a, Sphk1, Hcls1, } \\
\text { Clec9a, Flt3, Chil1, Zap70, Fgfr4, Casp1, Il27ra, Nefl, Nr1h4, Chia1, Tle6, Cbln1, Mmp9, } \\
\text { Dhx58, C3, Sema7a, Nfam1, Foxo6, Spry1, Pkdcc, Zfp219, Ptn, Fezf2, Inhbb, Ccr1, Apoe, } \\
\text { Gsdmd, Calca, Tbx2, Nkx2-2, Scx, Ccr2, Ctgf, Cebpd, Rnf207, Irf8, Fgfr3, Hpn, Adrb2, } \\
\text { Cd27, Cebpb, Cd3e, Bmp6, Camp, Ngfr, Adm, Tbx18, Rgs14, Icam1, Trim15, Foxd1, Cd40, } \\
\text { Casp4, Plk5, Gpr37l1, Ccl2, Gfap, Egr3, Lrg1, Alox12b, Vtcn1, Lrrc4b, Mapk13, Fst, Dll3, } \\
\text { Ltk, Smad6, Pdgfa, Prom1, Atoh8, Tbx1, Ltf, Ltb, Ccdc88b, Bmp2, Hap1, Fgr, Card9, } \\
\text { Unc93b1, Smo, Id4, Wnt6, Agrn, Selp, Metrn, Zc3h12a, Ascl1, Cd1d1, Nell1, Sox17, } \\
\text { Afap112, Scn1b, Sox2, Alox5, Cebpa, Fzd9, Pawr, Ltbp3, Adra2a, Adgrb1, Mmp14, P2ry2, } \\
\text { S100a9, Irx3, Nfatc4, Card11, Chga, Gpr68, Islr2, and Zic2 }\end{array}$ \\
\hline
\end{tabular}




\begin{tabular}{|c|c|c|c|c|}
\hline $\begin{array}{l}\text { GO:0006928 movement of cell or } \\
\text { subcellular component }\end{array}$ & 138 & 16.29 & $3.14 \mathrm{E}-16$ & $\begin{array}{l}\text { Cxcl1, Sema4c, Elane, Foxc2, Myh14, Uty, Tekt2, Egr1, Gata2, Vav2, Ccl7, Ccl19, Svs2, } \\
\text { Ptger4, Ptk7, Etv4, Spns2, Kifc3, Cxcl5, Ephb3, Sphk1, Kiss1r, Arhgap4, Vax1, Fgfr4, } \\
\text { Nr4a1, Nefl, Mmp9, Igsf8, Sox18, Sema7a, P2ry6, St14, Kif19a, Ptn, Gpr35, Dnaic2, Fezf2, } \\
\text { Nrtn, Podxl2, Sox1, Apoe, Ccr1, Styk1, Calca, Cfap53, Selplg, Fpr2, Ccr2, Ctgf, Rnf207, } \\
\text { Sema5b, Pstpip1, Adam15, Ngfr, Isl2, Sema3b, Icam1, Kif12, Foxd1, Lamb2, Matk, Nexn, } \\
\text { Cldn7, Wwc1, Ccl2, Apc2, Kif21b, Efnb3, Atn1, Sema6b, Egr3, Cdk5r2, Snta1, Dapk3, Bin2, } \\
\text { Itgam, Myo1g, Itga2b, Cep131, Arc, Tyro3, Pdgfa, Atoh8, Tbx1, Lhx2, Col18a1, Plekhg5, } \\
\text { Bmp2, Hap1, Fgr, Klc3, Svs3a, Asap3, Smo, Coro1a, Cfap73, Amica1, Sema3f, Mnx1, } \\
\text { Fmnl1, Agrn, Selp, Ccl6, Sell, S100a8, Atp1a2, Zc3h12a, Ascl1, Nck2, Itgb7, Sox17, } \\
\text { Sema6c, Scn1b, Wnt5b, Runx3, Rhbdf1, Pawr, Jak3, Adra2a, Six2, Gas1, Sema3g, } \\
\text { Mmp14, P2ry2, Trem3, Dpysl4, Saa3, Nkx2-1, S100a9, Retnlg, Tpbgl, Svs3b, Kif7, } \\
\text { Spock1, Chga, Kif26a, and Zic2 }\end{array}$ \\
\hline GO:0040011 locomotion & 127 & 14.99 & 4.88E-16 & $\begin{array}{l}\text { Cxcl1, Sema4c, Elane, Foxc2, Tekt2, Egr1, Gata2, Vav2, Ccl7, Ccl19, Svs2, Ptger4, Ptk7, } \\
\text { Etv4, Spns2, Nlgn2, Cxcl5, Ephb3, Sphk1, Arhgap4, Kiss1r, Vax1, Fgfr4, Nr4a1, Nefl, } \\
\text { Mmp9, Sox18, Igsf8, Sema7a, P2ry6, St14, Ptn, Gpr35, Fezf2, Nrtn, Sox1, Podxl2, Apoe, } \\
\text { Ccr1, Styk1, Calca, Selplg, Fpr2, Ccr2, Ctgf, Sema5b, Pstpip1, Adam15, Ngfr, Tymp, Isl2, } \\
\text { Sema3b, Icam1, Foxd1, Lamb2, Nexn, Matk, Cldn7, Wwc1, Ccl2, Apc2, Efnb3, Atn1, } \\
\text { Sema6b, Egr3, Cdk5r2, Dapk3, Bin2, Itgam, Myo1g, Itga2b, Arc, Tyro3, Pdgfa, Atoh8, } \\
\text { Tbx1, Lhx2, Col18a1, Plekhg5, Bmp2, Fgr, Svs3a, Asap3, Smo, Coro1a, Amica1, Semaf,, } \\
\text { Mnx1, Fmnl1, Agrn, Selp, Ccl6, Sell, S100a8, Atp1a2, Zc3h12a, Ascl1, Nck2, Itgb7, Sox17, } \\
\text { Sema6c, Scn1b, Wnt5b, Runx3, Cxcr6, Rhbdf1, Pawr, Jak3, Adra2a, Six2, Gas1, Mmp14, } \\
\text { Sema3g, Ackr3, P2ry2, Trem3, Dpysl4, Saa3, Nkx2-1, S100a9, Retnlg, Tpbgl, Svs3b, } \\
\text { Spock1, Chga, Kif26a, and Zic2 }\end{array}$ \\
\hline $\begin{array}{l}\text { GO:0050793 regulation of } \\
\text { developmental process }\end{array}$ & 166 & 19.59 & $2.16 \mathrm{E}-15$ & $\begin{array}{l}\text { Myh4, Cxcl1, Cdkn1c, Sema4c, Foxc2, Myh14, Rbm38, Hes7, Nab2, Egr1, Trpv2, Gata2, } \\
\text { Ngef, Zfpm1, Gli1, Ccl19, Ccl7, Dll1, Ptger4, Ptk7, Trp73, Etv4, Hmga1, Nlgn2, Tead3, Shb, } \\
\text { Cxcl5, Ephb3, Stk11, Hlx, Chadl, Cldn5, Il4ra, Sphk1, Hcls1, Flt3, Chil1, Arhgap4, Vax1, } \\
\text { Zap70, Fgfr4, Maff, Il27ra, Nefl, Nr1h4, Tle6, Cbln1, Mmp9, Sox13, Dusp6, C3, Sema7a, } \\
\text { Errfi1, Nfam1, Foxo6, Spry1, Pkdcc, Zfp219, Ptn, Fezf2, Ccr1, Apoe, Nkx3-2, Tbx2, Nkx2-2, } \\
\text { Ccr2, Scx, Ctgf, Fgfr3, Hpn, Adrb2, Cd27, Cebpb, Sema5b, Nfe2, Cd3e, Bmp6, Camp, } \\
\text { Ngfr, Adm, Isl2, Sema3b, Fzd1, Tbx18, Rgs14, Icam1, Foxd1, Cd40, Mycl, Aatk, Mir212, } \\
\text { Hck, Plk5, Gpr37l1, Lfng, Chad, Wwc1, Ccl2, Sema6b, Gfap, Egr3, Lrg1, Dapk3, Lrrc4b, } \\
\text { Pi16, Fst, Dll3, Arc, Ltk, Spr, Pglyrp1, Ngp, Pdgfa, Prom1, Atoh8, Tbx1, Ltf, Bmp2, Hap1, } \\
\text { Fgr, Ankrd6, Smo, Sfrp5, Coro1a, Id4, Sema3f, Wnt6, Fmnl1, Agrn, Mir132, Metrn, Colq, } \\
\text { Zc3h12a, Ascl1, Rhou, Id3, Cd1d1, Nell1, Sox17, Sema6c, Scn1b, Sox2, Adamts7, Fzd9, } \\
\text { Wtip, Pawr, Ltbp3, Jak3, E4f1, Six2, Lingo1, Spi1, Adgrb1, Dact3, Sema3g, Mmp14, P2ry2, } \\
\text { Nkx2-1, Irx3, Tpbgl, Nfatc4, Card11, Spock1, Gpr68, Islr2, and Zic2 }\end{array}$ \\
\hline GO:0002376 immune system process & 166 & 19.59 & $1.16 \mathrm{E}-14$ & $\begin{array}{l}\text { Sppl2b, Cxcl1, Cdkn1c, Elane, Egr1, Gata2, Zfpm1, Klf2, Jag2, Ccl19, Ccl7, Dll1, Ptger4, } \\
\text { Spns2, H2-Ab1, Lyl1, Shb, Cd79a, Cxcl5, Spn, Ephb3, Stk11, Lrmp, Hlx, Il18r1, Lat2, Il4ra, } \\
\text { Tbc1d10c, Clec5a, Hcls1, Flt3, Lat, Zap70, Casp1, Il27ra, Rab33a, Nr1h4, Chia1, Mmp9, } \\
\text { Crlf2, Sox13, Junb, Dhx58, C3, Sema7a, Nfam1, Lcn2, Icos, Gpr35, Pigr, Ccr1, Podxl2, } \\
\text { Gsdmd, Styk1, Relb, Calca, Selplg, C7, Nkx3-2, Blk, Fpr2, Ccr2, Cebpd, Irf8, Fgfr3, H2- } \\
\text { Q10, Cd300lf, Cebpb, Cd27, Cactin, Pstpip1, Il3ra, Cd3e, Bmp6, Adam15, Camp, Ngfr, } \\
\text { Cd3d, Adm, Icam1, Trim15, Cd40, Clec4d, Htra1, Ctsg, Cfb, Casp4, Hck, Matk, Sh2b2, } \\
\text { Lfng, Cldn7, Rasal3, Ccl2, Btk, Igfbp2, Efnb3, Zc3h12d, Egr3, H2-Q1, Pla2g2f, Ctse, } \\
\text { Pmaip1, Dapk3, Vtcn1, Itgam, Itgax, Myo1g, Itga2b, Sbn02, Fst, Cspg5, Smad6, Pglyrp1, } \\
\text { Tyro3, Tbx1, Ltf, Ltb, Ccdc88b, Asb2, Fgr, Card9, Bst1, Unc93b1, Slpi, Coro1a, H2-Eb1, } \\
\text { Amica1, Tnfaip8l2, Pirb, Selp, Jchain, Ccl6, Sell, S100a8, Zc3h12a, Nck2, Fcgr2b, Itgb7, } \\
\text { Cd1d1, Gch1, Runx3, Cebpa, Tnfrsf25, Fzd9, Hp, Pawr, Jak3, Spi1, Mpo, Treml2, Mmp14, } \\
\text { Ackr3, Trem3, Ephb6, S100a9, Retnlg, C1ra, Prtn3, Card11, Padi4, Kdm5d, Chga, C4b, } \\
\text { Gpr68, and Ncf1 }\end{array}$ \\
\hline
\end{tabular}


Table 5. Continued

\begin{tabular}{|c|c|c|c|c|}
\hline GO:0001816 cytokine production & 67 & 7.91 & $1.02 \mathrm{E}-13$ & $\begin{array}{l}\text { Elane, Egr1, Vtcn1, Klf2, Zfpm1, Dll1, Ccl19, Mapk13, Ptger4, Pglyrp1, Ltf, Cxcl5, Spn, Ltb, } \\
\text { Ccdc88b, Fgr, Card9, Il18r1, Sphk1, Il4ra, Unc93b1, Clec5a, Clec9a, Chil1, Ackr1, Flt3, } \\
\text { Fgfr4, Casp1, Il27ra, Nr1h4, Chia1, Crlf2, Dhx58, C3, Sema7a, Errfi1, Zc3h12a, Nfam1, } \\
\text { Fcgr2b, Cd1d1, Afap112, Inhbb, Gsdmd, Relb, Runx3, Ccr2, Ltbp3, Pawr, Irf8, Jak3, } \\
\text { Adra2a, Cebpb, Cd27, Cactin, Cd3e, Pomc, P2ry2, Trem3, Trim15, Cd40, Ephb6, Casp4, } \\
\text { Nfatc4, Card11, Chga, Ccl2, and Btk }\end{array}$ \\
\hline GO:0044699 single-organism process & 626 & 73.90 & $1.38 \mathrm{E}-13$ & 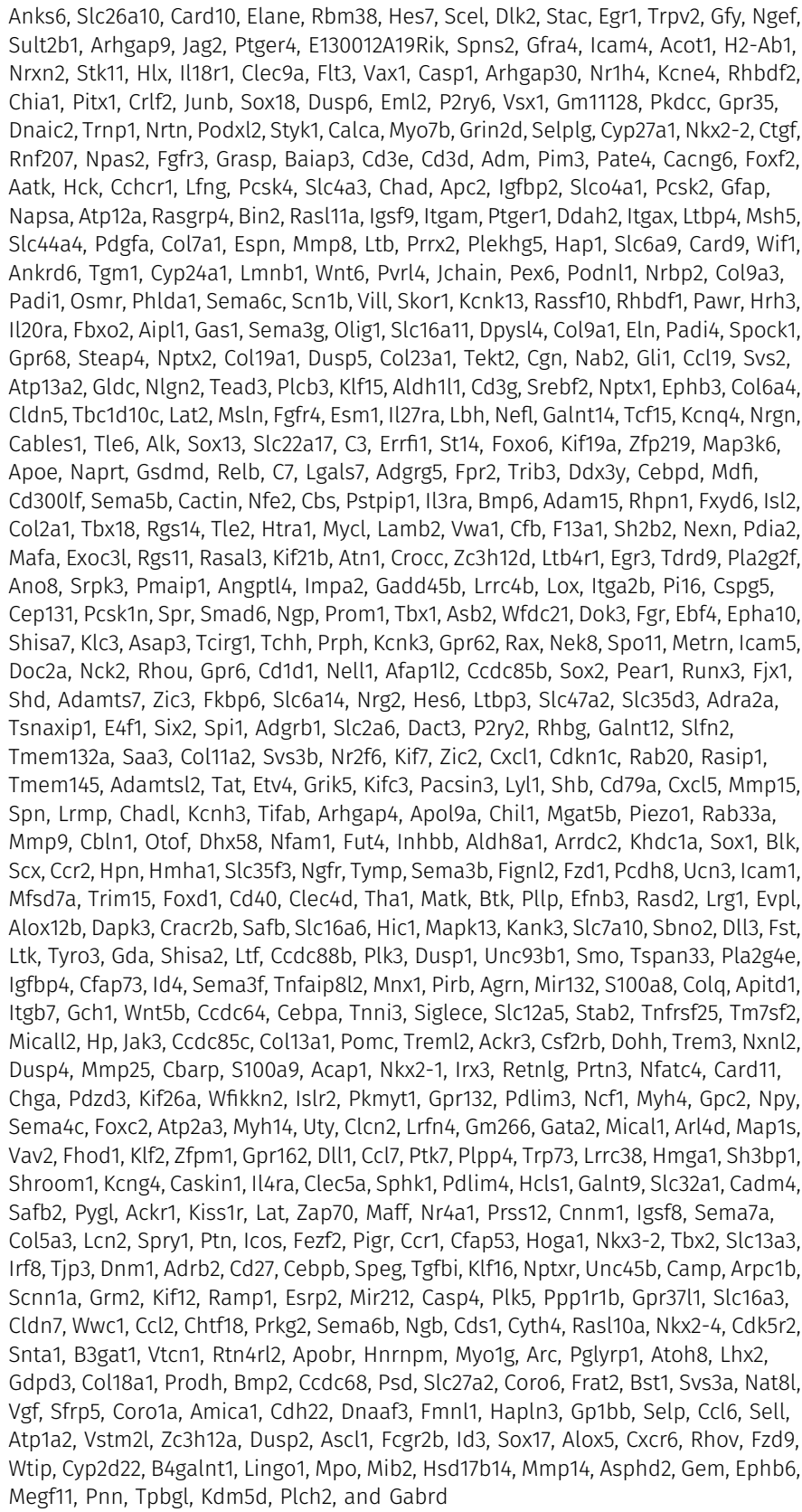 \\
\hline
\end{tabular}

(Continued on following page) 


\begin{tabular}{|c|c|c|c|c|}
\hline GO:0009888 tissue development & 139 & 16.41 & $\begin{array}{l}2.187987473633545 E- \\
13\end{array}$ & $\begin{array}{l}\text { Myh4, Cdkn1c, Sema4c, Rasip1, Foxc2, Myh14, Dusp5, Hes7, Cgn, Scel, Egr1, Gata2, } \\
\text { Zfpm1, Gli1, Klf2, Jag2, Dll1, Ptk7, Adamtsl2, Trp73, Etv4, Klf15, Mmp15, Hlx, Chadl, } \\
\text { Cldn5, Safb2, Vax1, Fgfr4, Maff, Nr4a1, Nr1h4, Tcf15, Mmp9, Pitx1, Junb, Sox18, Dusp6, } \\
\text { Sema7a, Errfi1, St14, Pkdcc, Zfp219, Spry1, Ptn, Nrtn, Ccr1, Nkx3-2, Tbx2, Nkx2-2, Scx, } \\
\text { Ctgf, Fgfr3, Hpn, Cebpb, Adrb2, Sema5b, Speg, Nfe2, Tgfbi, Cbs, Bmp6, Adam15, Ngfr,, } \\
\text { Adm, Col2a1, Sema3b, Fzd1, Tbx18, Pcdh8, Icam1, Foxd1, Esrp2, Mycl, Lamb2, Foxf2, } \\
\text { Nexn, Lfng, Sema6b, Srpk3, Evpl, Pi16, Sbno2, Fst, Dll3, Arc, Smad6, Col7a1, Pdgfa, } \\
\text { Prom1, Atoh8, Tbx1, Ltf, Lhx2, Mmp8, Col18a1, Prrx2, Asb2, Bmp2, Dusp1, Ankrd6, Smo, } \\
\text { Tgm1, Sfrp5, Id4, Sema3f, Mnx1, Wnt6, Dusp2, Ascl1, Id3, Sox17, Nell1, Sema6c, Sox2, } \\
\text { Wnt5b, Runx3, Tnni3, Zic3, Adamts7, Fzd9, Pawr, Ltbp3, Six2, Spi1, Gas1, Dact3, Mmp14, } \\
\text { Sema3g, Dusp4, Nkx2-1, Col9a1, Irx3, Col11a2, Nfatc4, Eln, Col19a1, Pdlim3, and Zic2 }\end{array}$ \\
\hline $\begin{array}{l}\text { GO:0048583 regulation of response to } \\
\text { stimulus }\end{array}$ & 209 & 24.67 & 3.267E-13 & 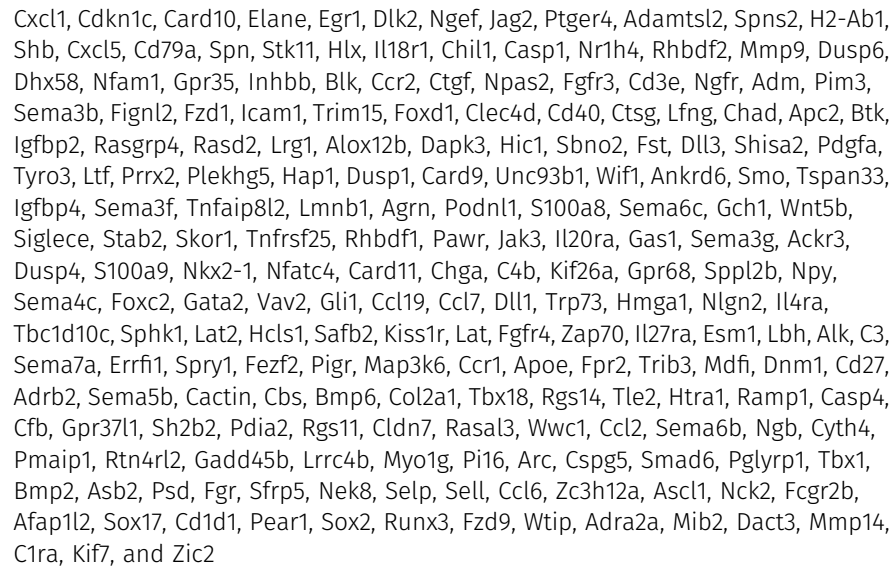 \\
\hline
\end{tabular}

Whereas multiple links between gut microbiota and the hypothalamic-pituitary axis have been reported (Appleton, 2018), our results constitute the very first evidence for the involvement of a chemokine receptor, $\mathrm{Cxcr} 2$, in the control of pituitary function and the reproductive system and that this phenomenon is dependent on microbiota (Fig 10). Although there have been no reports of any action of $\mathrm{Cxcr} 2$ in pituitary function till date, $\mathrm{Cxcr} 2$ has been shown to be important for the positioning of oligodendrocyte precursors in the spinal cord (Tsai et al, 2002) and in myelin repair (Liu et al, 2010), but overall, the effects of $\mathrm{Cxcr} 2$ in central nervous system are poorly understood. On the other hand, a few reports have suggested a potential role for Cxcr2 in controlling the effects of microbiota. Indeed, it was shown that microbiota promotes the recruitment of Cxcr2-positive neutrophils that can protect from amebic colitis (Watanabe et al, 2017). Finally, so far, no direct evidence of a role of Cxcr2 in the control of reproduction has been reported. Some reports suggest that $\mathrm{Cxcr} 2$ might contribute to the development of preeclampsia (Wu et al, 2016; Chen et al, 2019) and in the control, pregnancy tolerance through neutrophils (Kang et al, 2016). In addition, loss of $\mathrm{Cxc} 2$ ligand has been shown to result in premature senescence in placenta-derived mesenchymal stem cells ( $\mathrm{Li}$ et al, 2017).

In summary, our results highlight a unique unexplored role for Cxcr2 in pituitary and reproductive function that is dependent on chronic infections (summarized in Fig 10). These findings reinforce an imperative; to be translationally relevant, the use of animal models to understand disease mechanisms must take into account animal husbandry conditions and disease phenotyping requiring the absence or presence of chronic infections.

\section{Materials and Methods}

\section{Animal models and housing}

All animal experiments conform to our animal protocols that were reviewed and approved by the Institutional Animal Care and Use Committee. Cxcr2- / - mice (Cacalano et al, 1994) obtained from the Jackson Laboratory were in BALB/c genetic background as well as their littermate control (WT) mice. Mice were either housed in an SOPF facility or transferred to a conventional animal facility. To favor the acquisition of microbiota from conventional facility to the SOPF animals which were transferred to conventional conditions, the bedding of conventional animals was added to one of the transferred SOPF animals during the first weeks. The phenotype of the animals housed in the conventional facility was first analyzed following at least one or two generations of breeding to enable the establishment of bystander infections in all animals. Because of the reduced fertility of Cxcr2-/- animals in conventional conditions, homozygous animals were obtained by crossing heterozygous animals.

\section{Pathogen screening}

The presence of potential infections was monitored by Charles River Research Animal Diagnostic Services on whole animals or body swabs, oral swabs, and feces of the animals according to PCR rodent infectious agent (PRIA) instructions. The main bystander infections observed are shown in Fig S1. 
Table 6. Most enriched pathways for genes down-regulated in the pituitary of KO versus WT conventional animals.

\begin{tabular}{|c|c|c|c|c|}
\hline GO biological process term & Count & $\%$ & $P$-value & Genes \\
\hline $\begin{array}{l}\text { GO:0007156 homophilic cell adhesion via plasma } \\
\text { membrane adhesion molecules }\end{array}$ & 40 & 5.01 & $6.11 \mathrm{E}-23$ & $\begin{array}{l}\text { Pcdhga10, Pcdhga12, Pcdha11, Pcdhac2, Pcdha12, Pcdhb6, } \\
\text { Pcdhb11, Pcdhgb1, Pcdhgc5, Pcdhb21, Pcdh10, Pcdhga9, } \\
\text { Pcdhb8, Pcdha8, Pcdhga5, Pcdhga3, Pcdhgb8, Pcdhgb7, } \\
\text { Pcdhgb5, Pcdhac1, Igsf9b, Pcdha9, Pcdhgb4, Pcdhgb2, } \\
\text { Pcdhga8, Pcdhga6, Cdh12, Pcdhb19, Pcdhgb6, Cdh18, } \\
\text { Pcdhb2, Pcdhb1, Pcdhb16, Pcdhga7, Sdk2, Cdh7, Pcdh11x, } \\
\text { Pcdhga4, Pcdha5, and Pcdhga11 }\end{array}$ \\
\hline $\begin{array}{l}\text { GO:0045653 negative regulation of megakaryocyte } \\
\text { differentiation }\end{array}$ & 9 & 1.12 & $2.35 \mathrm{E}-8$ & $\begin{array}{l}\text { Hist1h4a, Hist1h4c, Hist1h4i, Hist1h4f, Hist1h4j, Hist1h4d, } \\
\text { Hist1h4h, Hist1h4k, and Hist2h4 }\end{array}$ \\
\hline GO:0006811 ion transport & 78 & 9.77 & $1.28 \mathrm{E}-6$ & $\begin{array}{l}\text { Slc36a4, G6pdx, Ano4, Vip, Ank1, Nkain3, Tspan13, Mif, Ucp1, } \\
\text { Atp5g1, Slc15a1, Lrrc8b, Utrn, Kcnv2, Cd84, Grin2a, Slc24a2, } \\
\text { Atp7b, Slc17a9, Ryr2, Clcn5, Kcnk9, Cacna1c, Kcna6, Kcnd3, } \\
\text { Slc1a7, Ano5, Gabrb2, Slc4a5, Asic2, Kcnip1, Kcna3, Nos1, } \\
\text { Cacna1g, Crhbp, Slc30a7, Kcnb2, Trpc6, Htr1b, Gabra1, Slc8a1, } \\
\text { Wnk3, Slc41a2, Oprk1, Mrs2, Akap6, Slc30a8, Kcns2, Slc16a7, } \\
\text { Kcnj6, Gal, Slc26a2, Atp6ap1l, Pla2r1, Klhl3, Cacna1e, Kcnf1, } \\
\text { Grid2, Kcnh5, Steap3, Tmem266, Aqp7, Slc5a8, Slc6a18, } \\
\text { Nos1ap, Slc7a7, Slc5a7, Scnn1g, Grin2b, Reln, Best3, Klhl24, } \\
\text { Nkain2, Chrna9, Slc8a3, Slc9a7, Cckar, and Scn10a }\end{array}$ \\
\hline GO:0030001 metal ion transport & 50 & 6.26 & $1.22 \mathrm{E}-5$ & $\begin{array}{l}\text { Cacna19, Slc30a7, Kcnb2, Trpc6, G6pdx, Htr1b, Vip, Slc8a1, } \\
\text { Nkain3, Tspan13, Wnk3, Mif, Slc41a2, Oprk1, Akap6, Mrs2, } \\
\text { Slc30a8, Utrn, Kcnv2, Grin2a, Cd84, Slc24a2, Kcns2, Atp7b, } \\
\text { Kcnj6, Gal, Ryr2, Klhl3, Kcnk9, Cacna1c, Cacna1e, Kcnf1, } \\
\text { Kcnh5, Steap3, Kcna6, Slc5a8, Kcnd3, Nos1ap, Slc5a7, } \\
\text { Scnn1g, Grin2b, Nkain2, Asic2, Slc8a3, Kcnip1, Kcna3, Nos1, } \\
\text { Slc9a7, Cckar, and Scn10a }\end{array}$ \\
\hline G0:0006812 cation transport & 55 & 6.89 & $1.76 \mathrm{E}-5$ & $\begin{array}{l}\text { G6pdx, Vip, Ank1, Nkain3, Tspan13, Mif, Ucp1, Atp5g1, Kcnv2, } \\
\text { Utrn, Cd84, Grin2a, Slc24a2, Atp7b, Ryr2, Kcnk9, Cacna1c, } \\
\text { Kcna6, Kcnd3, Asic2, Kcnip1, Nos1, Kcna3, Cacna19, Slc30a7, } \\
\text { Kcnb2, Trpc6, Htr1b, Slc8a1, Slc41a2, Wnk3, Oprk1, Mrs2, } \\
\text { Akap6, Slc30a8, Kcns2, Gal, Kcnj6, Klhl3, Atp6ap1l, Cacna1e, } \\
\text { Kcnf1, Kcnh5, Steap3, Slc5a8, Nos1ap, Slc5a7, Scnn1g, Grin2b, } \\
\text { Nkain2, Chrna9, Slc8a3, Slc9a7, Scn10a, and Cckar }\end{array}$ \\
\hline
\end{tabular}

(Continued on following page) 


\begin{tabular}{|c|c|c|c|c|}
\hline GO:0043269 regulation of ion transport & 40 & 5.01 & 1.97E-5 & $\begin{array}{l}\text { Crhbp, Cacna1g, Kcnb2, Trpc6, G6pdx, Vip, Slc8a1, Nkain3, } \\
\text { Tspan13, Wnk3, Mif, Oprk1, Slc15a1, Akap6, Utrn, Kcnv2, Cd84, } \\
\text { Kcns2, Ryr2, Gal, Kcnj6, Pla2r1, Cacna1c, Cacna1e, Kcnf1, } \\
\text { Kcnh5, Kcna6, Kcnd3, Nos1ap, Grin2b, Reln, Best3, Nkain2, } \\
\text { Klhl24, Asic2, Kcnip1, Kcna3, Nos1, Scn10a, Cckar }\end{array}$ \\
\hline GO:0015672 monovalent inorganic cation transport & 32 & 4.01 & $2.53 \mathrm{E}-5$ & $\begin{array}{l}\text { Kcnb2, Vip, Ank1, Slc8a1, Wnk3, Mif, Ucp1, Atp5g1, Oprk1, } \\
\text { Akap6, Utrn, Kcnv2, Kcns2, Kcnj6, Gal, Klhl3, Atp6ap1l, Kcnk9, } \\
\text { Kcnf1, Kcnh5, Kcna6, Slc5a8, Kcnd3, Slc5a7, Scnn1g, Asic2, } \\
\text { Slc8a3, Kcnip1, Kcna3, Nos1, Slc9a7, and Scn10a }\end{array}$ \\
\hline GO:0007155 cell adhesion & 81 & 10.15 & $5.98 \mathrm{E}-5$ & $\begin{array}{l}\text { Pcdha11, Hist1h3e, Il2ra, Lamb3, Lpp, Itga1, Crisp2, Pcdhb11, } \\
\text { Cntnap5c, Pcdhgc5, Utrn, Cd84, Tenm1, Pcdhb21, Pcdhga9, } \\
\text { Tnr, Pcdha8, Pcdhga3, Nrxn3, Pcdhgb5, Igsf9b, Pcdha9, } \\
\text { Pcdhgb4, Adam12, Cyfip2, Pkhd1, Hapln4, Pcdhb2, Edil3, } \\
\text { Pcdhb16, Prkdc, Pcdhga7, Cdh7, Pcdh11x, Snai2, Agr2, } \\
\text { Pcdhga4, Stat5a, Pcdhga10, Pcdhga12, Ncam2, Pcdhac2, } \\
\text { Pcdha12, Pcdhb6, Omg, Pcdhgb1, Plet1, Scgb1a1, Omd, } \\
\text { Cgref1, Crebbp, Pcdh10, Itgb8, Pcdhb8, Rps26, Pcdhga5, } \\
\text { Pcdhgb8, Pcdhgb7, Sned1, Pcdhac1, Grid2, Gpam, Pcdhgb2, } \\
\text { Pcdhga8, Epha5, Cntnap5b, Pcdhb19, Cdh12, Pcdhgb6, } \\
\text { Pcdhga6, Cdh18, Hapln1, Pcdhb1, Tcam1, Reln, Sdk2, Phldb2, } \\
\text { Flrt1, Pcdha5, S100a10, and Pcdhga11 }\end{array}$ \\
\hline G0:0034762 regulation of transmembrane transport & 30 & 3.75 & $6.62 \mathrm{E}-5$ & $\begin{array}{l}\text { Crhbp, Kcnb2, Trpc6, G6pdx, Slc8a1, Tspan13, Wnk3, Oprk1, } \\
\text { Akap6, Utrn, Kcnv2, Kcns2, Kcnj6, Gal, Ryr2, Cacna1c, } \\
\text { Cacna1e, Kcnf1, Kcnh5, Kcna6, Kcnd3, Nos1ap, Reln, Klhl24, } \\
\text { Asic2, Oaz3, Kcnip1, Nos1, Kcna3, and Scn10a }\end{array}$ \\
\hline GO:0098655 cation transmembrane transport & 36 & 4.51 & $7.31 \mathrm{E}-5$ & $\begin{array}{l}\text { Cacna1g, Kcnb2, Trpc6, Htr1b, G6pdx, Slc8a1, Tspan13, Wnk3, } \\
\text { Atp5g1, Oprk1, Akap6, Utrn, Kcnv2, Slc24a2, Kcns2, Atp7b, } \\
\text { Ryr2, Kcnj6, Gal, Atp6ap1l, Kcnk9, Cacna1c, Cacna1e, Kcnf1, } \\
\text { Kcnh5, Kcna6, Kcnd3, Nos1ap, Scnn1g, Asic2, Slc8a3, Kcnip1, } \\
\text { Kcna3, Nos1, Slc9a7, and Scn10a }\end{array}$ \\
\hline GO:0034765 regulation of ion transmembrane transport & 29 & 3.63 & 7.69E-5 & $\begin{array}{l}\text { Crhbp, Kcnb2, Trpc6, G6pdx, Slc8a1, Tspan13, Wnk3, Oprk1, } \\
\text { Akap6, Utrn, Kcnv2, Kcns2, Kcnj6, Gal, Ryr2, Cacna1c, } \\
\text { Cacna1e, Kcnf1, Kcnh5, Kcna6, Kcnd3, Nos1ap, Reln, Klhl24, } \\
\text { Asic2, Kcnip1, Nos1, Kcna3, and Scn10a }\end{array}$ \\
\hline GO:0098662 inorganic cation transmembrane transport & 34 & 4.26 & 1.06E-4 & $\begin{array}{l}\text { Cacna1g, Kcnb2, Trpc6, Htr1b, G6pdx, Slc8a1, Wnk3, Atp5g1, } \\
\text { Oprk1, Akap6, Utrn, Kcnv2, Slc24a2, Kcns2, Atp7b, Ryr2, Kcnj6, } \\
\text { Gal, Atp6ap1l, Kcnk9, Cacna1c, Cacna1e, Kcnf1, Kcnh5, Kcna6, } \\
\text { Kcnd3, Nos1ap, Scnn1g, Slc8a3, Kcnip1, Kcna3, Nos1, Slc9a7, } \\
\text { and Scn10a }\end{array}$ \\
\hline GO:0007268 chemical synaptic transmission & 37 & 4.63 & $1.35 \mathrm{E}-4$ & $\begin{array}{l}\text { Crhbp, Cacna1g, Ptpn5, Htr1b, Gabra1, Sytl5, Ssh1, Lrrtm2, } \\
\text { Oprk1, Grin2a, Slc24a2, Cartpt, Cplx1, Syt6, Sstr2, Tnr, Rims4, } \\
\text { Cacna1c, Nrxn3, Tmod2, Pclo, Igsf9b, Grid2, Gpr149, Slc5a7, } \\
\text { Grin2b, Pcdhb16, Reln, Gabrb2, Chrna9, Gpr21, Adnp, Slc8a3, } \\
\text { Slitrk5, Grm5, Nos1, and Retn }\end{array}$ \\
\hline
\end{tabular}


Table 6. Continued

\begin{tabular}{|c|c|c|c|c|}
\hline GO:0099536 synaptic signaling & 37 & 4.63 & $1.35 \mathrm{E}-4$ & $\begin{array}{l}\text { Crhbp, Cacna1g, Ptpn5, Htr1b, Gabra1, Syt15, Ssh1, Lrrtm2, } \\
\text { Oprk1, Grin2a, Slc24a2, Cartpt, Cplx1, Syt6, Sstr2, Tnr, Rims4, } \\
\text { Cacna1c, Nrxn3, Tmod2, Pclo, Igsf9b, Grid2, Gpr149, Slc5a7, } \\
\text { Grin2b, Pcdhb16, Reln, Gabrb2, Chrna9, Gpr21, Adnp, Slc8a3, } \\
\text { Slitrk5, Grm5, Nos1, and Retn }\end{array}$ \\
\hline GO:0099537 trans-synaptic signaling & 37 & 4.63 & $1.35 \mathrm{E}-4$ & $\begin{array}{l}\text { Crhbp, Cacna1g, Ptpn5, Htr1b, Gabra1, Sytl5, Ssh1, Lrrtm2, } \\
\text { Oprk1, Grin2a, Slc24a2, Cartpt, Cplx1, Syt6, Sstr2, Tnr, Rims4, } \\
\text { Cacna1c, Nrxn3, Tmod2, Pclo, Igsf9b, Grid2, Gpr149, Slc5a7, } \\
\text { Grin2b, Pcdhb16, Reln, Gabrb2, Chrna9, Gpr21, Adnp, Slc8a3, } \\
\text { Slitrk5, Grm5, Nos1, and Retn }\end{array}$ \\
\hline GO:0098916 anterograde trans-synaptic signaling & 37 & 4.63 & $1.35 \mathrm{E}-4$ & $\begin{array}{l}\text { Crhbp, Cacna1g, Ptpn5, Htr1b, Gabra1, Sytl5, Ssh1, Lrrtm2, } \\
\text { Oprk1, Grin2a, Slc24a2, Cartpt, Cplx1, Syt6, Sstr2, Tnr, Rims4, } \\
\text { Cacna1c, Nrxn3, Tmod2, Pclo, Igsf9b, Grid2, Gpr149, Slc5a7, } \\
\text { Grin2b, Pcdhb16, Reln, Gabrb2, Chrna9, Gpr21, Adnp, Slc8a3, } \\
\text { Slitrk5, Grm5, Nos1, and Retn }\end{array}$ \\
\hline GO:0048511 rhythmic process & 27 & 3.38 & $2.50 \mathrm{E}-4$ & $\begin{array}{l}\text { Prok2, Fox03, Setx, Oprk1, Pgr, Grin2a, Cartpt, Crebbp, Nhlh2, } \\
\text { Dmrta1, Prox1, Gpr149, Nrip1, Esr1, Arntl2, Atm, Prl, Grin2b, } \\
\text { Prkdc, Tyms, Rbm4, Alb, Uba52, Adnp, Retn, Mmp19, and } \\
\text { Stat5a }\end{array}$ \\
\hline GO:0006813 potassium ion transport & 18 & 2.25 & $3.85 \mathrm{E}-4$ & $\begin{array}{l}\text { Kenf1, Kcnh5, Kcnb2, Kcna6, Vip, Kcnd3, Mif, Oprk1, Akap6, } \\
\text { Kcnv2, Kcns2, Gal, Kcnj6, Kcnip1, Kcnk9, Nos1, Kcna3, and } \\
\text { Slc9a7 }\end{array}$ \\
\hline GO:0065008 regulation of biological quality & 145 & 18.17 & 4.09E-4 & $\begin{array}{l}\text { Slitrk3, Ano4, Foxo3, Vps13c, Vip, Frmpd4, Dock4, Lrrtm2, Mif, } \\
\text { Fgd4, Ryr2, Tnr, Kcnk9, Cacna1c, Nrxn3, Pkhd1, Kcnd3, Esr1, } \\
\text { Asic2, Agr2, Alb, Rbm4, Prlr, Slitrk5, Kcnip1, Nos1, Retn, } \\
\text { 4932438A13Rik, Crhbp, Ptpn5, Heg1, Oit1, Trpc6, Htr1b, } \\
\text { Plxna4, Sytl5, Stxbp5l, Dmxl1, Slc8a1, Ssh1, Wnk3, Tacr3, } \\
\text { Myrip, Omg, Palm2, Tmsb10, Scgb1a1, Slc30a8, Gal, Nop10, } \\
\text { Rims4, Cacna1e, Grid2, Kcnh5, Lnpep, Gpam, Pcsk6, Steap3, } \\
\text { Sesn3, Gnat2, Kdelr3, Slc5a7, Rcor1, Scnn1g, Sorl1, Neb, } \\
\text { Atp8b3, Usp13, Chrna9, Ptch1, Flrt1, Adnp, Grm5, Slc9a7, } \\
\text { Cckar, Ppargc1b, Gckr, G6pdx, Il2ra, Ank1, Pycr1, Itga1, Cela2a, } \\
\text { Grin2a, Brwd3, Slc24a2, Tenm1, Atp7b, Txndc2, Gng3, Vps13d, } \\
\text { Tmod2, Cbl, Igsf9b, Ptpro, Lyst, Vkorc1, Myl4, Chst8, Hmbox1, } \\
\text { Prl, Prkdc, Ar, Slc4a5, Tyms, Nr3c2, Gpr21, Mbd5, Stat5a, } \\
\text { Cacna1g, Slc30a7, Tnks, Scd1, Pak3, Oprk1, Akap6, Col1a2, } \\
\text { Cartpt, Cplx1, Syt6, Kcnj6, Slc26a2, Klhl3, Syndig1, Adgrb3, } \\
\text { Pclo, Nme2, Malat1, Epha5, Cdkl5, F8, Fstl4, Nrip1, Uprt, Aqp7, } \\
\text { Arhgap5, Rab38, Alms1, Atm, Ern1, Grin2b, Rab11fip2, Reln, } \\
\text { Slc8a3, and Scn10a }\end{array}$ \\
\hline GO:0007610 behavior & 41 & 5.13 & 4.32E-4 & $\begin{array}{l}\text { Crhbp, Ptpn5, Aff2, Htr1b, Vip, Mif, Tacr3, Oprk1, Hipk2, } \\
\text { Grin2a, Pgr, Slc24a2, Cartpt, Gal, Nhlh2, Tnr, Cacna1c, Nrxn3, } \\
\text { Dmrta1, Tmod2, Cacna1e, Adgrb3, Strn, Uba6, Grin2b, Prl, Ar, } \\
\text { Reln, Adcy8, Unc79, Alb, Gpr21, Adnp, Mbd5, Slitrk5, Slc8a3, } \\
\text { Pak7, Grm5, Nos1, Retn, and Cckar }\end{array}$ \\
\hline
\end{tabular}

\section{Analysis of estrus cycle}

Vaginal smears of WT and KO mice were performed in $\mathrm{NaCl}$ 9/1000, fixed in methanol, and then stained with Giemsa dye. Slides were scanned with a nanozoomer (Hamamatsu).

\section{Whole mounts of mammary glands}

Mammary glands were spread on glass slides and fixed in Carnoy's fixative for $4 \mathrm{~h}$. After rehydration, the glands were stained with carmine alum stain overnight. The glands were then cleared in xylene for several weeks to eliminate the fat.

\section{RNA extraction and reverse transcriptase, quantitative PCR}

Total RNA was isolated using TRIzol reagent (Thermo Fisher Scientific), as described by the manufacturer. Reverse transcription was performed with $1 \mu \mathrm{g}$ of total RNA using random primers and with M-MLV enzyme (Thermo Fisher Scientific). Real-time quantitative PCR was realized with SYBR green Master Mix (Roche), on a 

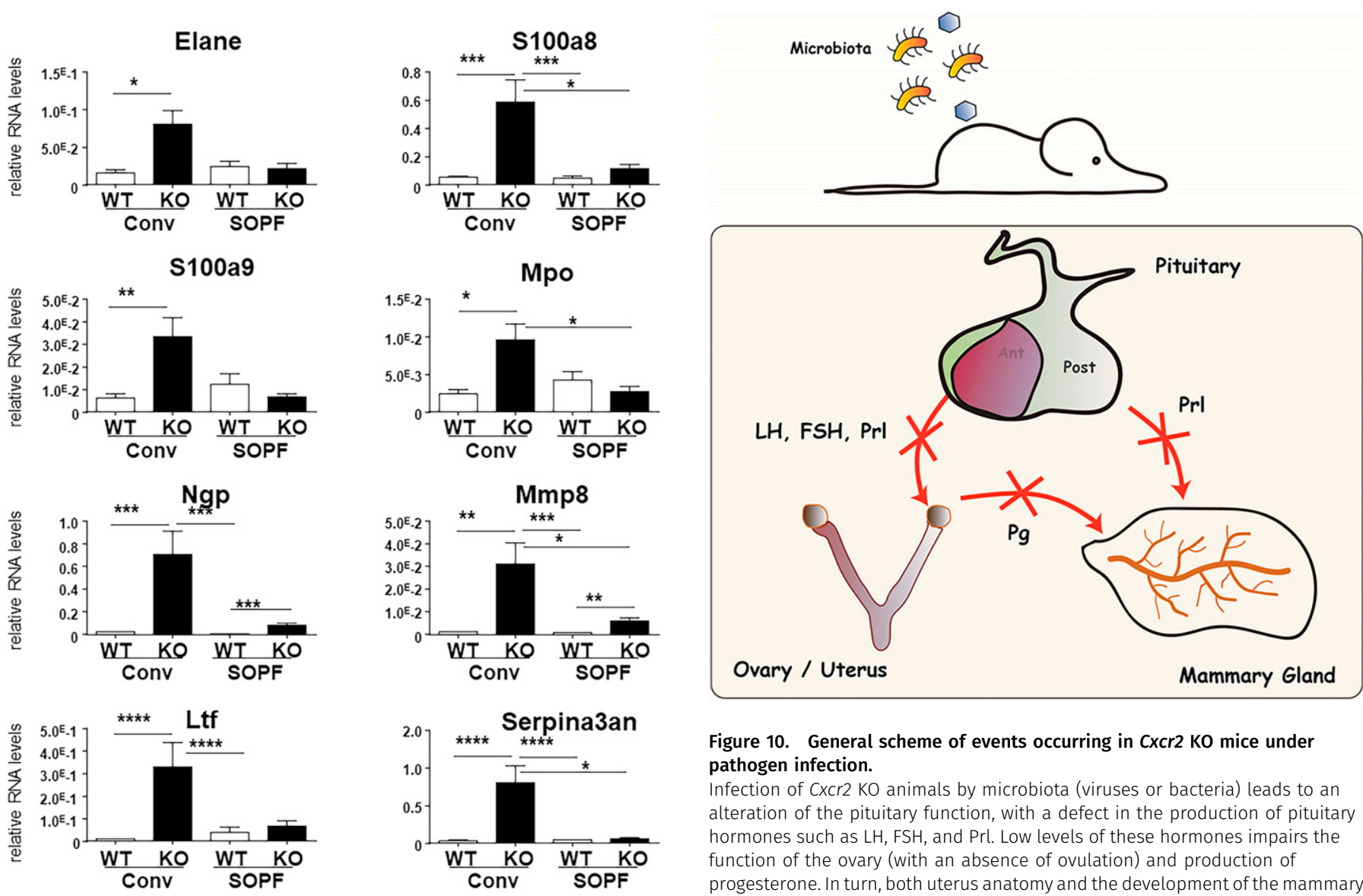

Figure 10. General scheme of events occurring in Cxcr2 KO mice under pathogen infection.

Infection of $\mathrm{CxCr} 2 \mathrm{KO}$ animals by microbiota (viruses or bacteria) leads to an alteration of the pituitary function, with a defect in the production of pituitary hormones such as LH, FSH, and Prl. Low levels of these hormones impairs the function of the ovary (with an absence of ovulation) and production of progesterone. In turn, both uterus anatomy and the development of the mammary gland are affected.
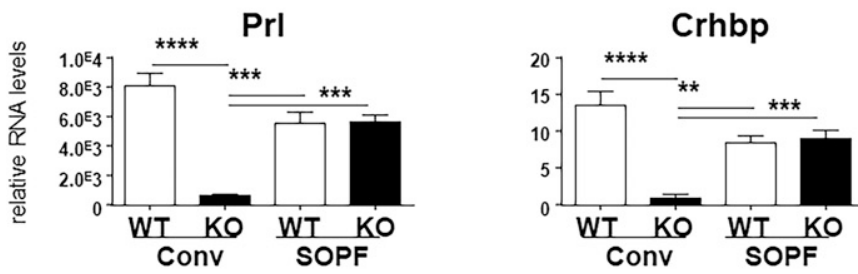

Light Cycler 480 instrument (Roche) as previously described (Escobar et al, 2015). Ribosomal protein S9 (rS9) and GAPDH were used as an internal control. The sequence of the primers used in this study is indicated in Table S2. Results are expressed as N-fold differences in target gene expression relative to the internal control gene and termed "mRNA expression," were determined as mRNA expression $=2^{-\Delta c t s a m p l e}$, where the $\Delta \mathrm{Ct}$ value of the sample was determined by subtracting the $\mathrm{Ct}$ value of the target gene from the $\mathrm{Ct}$ value of the average of the internal control genes. Target genes were considered to be non-detectable when the Ct value was above 35.

\section{RNAseq data processing}

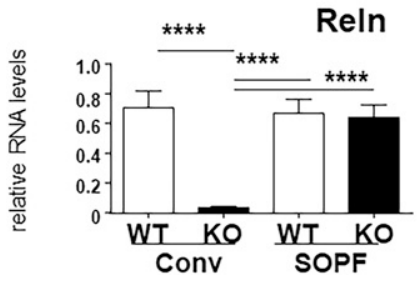

RNA integrity and quality was verified using RNA ScreenTape kit and Tapestation 2200 apparatus from AGILENT. cDNA libraries were synthesized using Sureselect Strand-Specific mRNA library prep (Agilent). Library quality was checked on Tapestation 2200 apparatus from AGILENT with DNA 1000 ScreenTape. Samples were sequenced on NextSeq 500 (Illumina) with an average sequencing depth of 30 millions of paired-end reads. Length of the reads was 75 bp. Each 12 Plex sample was sequenced on one HighOutput FlowCell ( $2 \times 400$ Millions of 75 bases reads). Raw sequencing data were quality-controlled with the FastQC program. Low-quality reads were trimmed or removed using Trimmomatic (minimum length: 40 
$\mathrm{bp}$ ). Reads were aligned to the mouse reference genome ( $\mathrm{mm} 10$ build) with the TopHat2 tool (option for no multi-hits). Mapping results were quality-checked using RNA-SeQC. Gene counts were obtained by read counting software featureCounts. Normalization and differential analysis were performed with the DESeq2 package with Benjamini-Hochberg false discovery rate multiple testing correction ( $P$-value $<0.05$; 1.5 -fold or higher change) comparing WT and $\mathrm{KO}$ animals. Clustering analysis was performed with Genesis software (Sturn et al, 2002). The data discussed in this publication have been deposited in NCBI's Gene Expression Omnibus (Edgar et al, 2002) and are accessible through GEO Series accession number GSE144231 (https://www.ncbi.nlm.nih.gov/geo/query/acc.cgi?acc=GSE144231).

\section{Hormone assessment}

Whole sera were used to measure the levels of estradiol and progesterone by Elisa with specific kits from Abnova. The levels of LH, FSH, GH, and PRL were assessed by luminex with Mouse Pituitary Magnetic Bead Panel (Merck Millipore).

\section{Ovary and mammary transplantations}

For ovary transplantations, WT or KO ovaries were obtained from 10wk-old donor mice. WT recipients were anesthetized with isoflurane and ovariectomized. One donor ovary was implanted in the recipient's ovarian bursa. After peritoneal and skin suture, recipients were allowed to recover for 2 wk. Ovaries were then analyzed at that stage or recipient mice were mated with WT males to evaluate fertility. For mammary gland transplantation, 12-wk-old WT and KO donor mice were used to obtain mammary glands. Small pieces of donor glands were implanted in the cleared mammary fat pad of one gland of 2.7-wk-old WT mice under general anesthesia. The contralateral mammary gland was cleared and kept as a control for clearing. Recipient mice were euthanized at $12 \mathrm{wk}$ and the transplanted mammary glands were analyzed by whole mount.

\section{Statistics}

Statistical analyses were carried out using unpaired Mann-Whitney test or when indicated Fisher exact test or Kruskal-Wallis test followed by Dunn's multiple comparisons test. To assess biological interpretation of the most differentially expressed genes, GO enrichment analysis was performed using the DAVID bioinformatics resources (https://david.ncifcrf.gov, Version 6.8).

\section{Data Availability}

The datasets discussed in this publication have been deposited in NCBI's Gene Expression Omnibus (Edgar et al, 2002) and are accessible through GEO Series accession number GSE144231 (https:/ / www.ncbi.nlm.nih.gov/geo/query/acc.cgi?acc=GSE144231).

\section{Supplementary Information}

Supplementary Information is available at https://doi.org/10.26508/lsa. 201900599.

\section{Acknowledgements}

This work was supported by Association pour la Recherche sur le Cancer (ARC) Foundation and la Ligue contre le Cancer to G Lazennec and by an Agence Nationale de la Recherche (ANR) grant 17-CE14-0019 to K Balabanian. We thank N Binart, A Richmond, and M Gary-Bobo for useful discussion and help in the experiments and $\mathrm{M} / \mathrm{L} /$ Loyalle and $\mathrm{A}$ Pulido for their help in mouse husbandry. We are grateful to J Stingl for training in mammary gland transplantation. We acknowledge the Plateau Central d'Elevage et d'Archivage (PCEA), Réseau des animaleries de Montpellier (RAM), Montpellier Ressources Imagerie (MRI), and Setiph facilities in Montpellier. We are grateful to Institut du cerveau et de la moëlle épinière in Paris for RNAseq experiments. We thank CECEMA-UM animal facility for housing and technical help. We are grateful to all master students who have contributed to this work.

\section{Authors Contributions}

C Timaxian: investigation.

I Raymond-Letron: investigation and methodology.

C Bouclier: investigation.

L Gulliver: investigation-review and editing.

L Le Corre: investigation.

$\mathrm{K}$ Chebli: investigation and methodology.

A Guillou: investigation.

P Mollard: writing-review and editing.

K Balabanian: writing-review and editing.

G Lazennec: conceptualization, supervision, investigation, methodology, and writing-original draft, review, and editing.

\section{Conflict of Interest Statement}

The authors declare that they have no conflict of interest.

\section{References}

Addison CL, Daniel TO, Burdick MD, Liu H, Ehlert JE, Xue YY, Buechi L, Walz A, Richmond A, Strieter RM (2000) The CXC chemokine receptor 2, CXCR2, is the putative receptor for ELR+CXC chemokine-induced angiogenic activity. J Immunol 165: 5269-5277. doi:10.4049/jimmunol.165.9.5269

Ali S, Lazennec G (2007) Chemokines: Novel targets for breast cancer metastasis. Cancer Metastasis Rev 26: 401-420. doi:10.1007/s10555007-9073-z

Appleton J (2018) The gut-brain axis: Influence of microbiota on mood and mental health. Integr Med 17: 28-32.

Bach JF (2002) The effect of infections on susceptibility to autoimmune and allergic diseases. N Engl J Med 347: 911-920. doi:10.1056/ nejmra020100

Bachelot A, Binart N (2005) Corpus luteum development: Lessons from genetic models in mice. Curr Top Dev Biol 68: 49-84. doi:10.1016/ s0070-2153(05)68003-9

Behringer R (2017) Mouse ovary transplantation. Cold Spring Harbor Protoc 2017. doi:10.1101/pdb.prot094458

Bellastella G, Maiorino MI, Bizzarro A, Giugliano D, Esposito K, Bellastella A, De Bellis A (2016) Revisitation of autoimmune hypophysitis: Knowledge and uncertainties on pathophysiological and clinical aspects. Pituitary 19: 625-642. doi:10.1007/s11102-016-0736-z

Beura LK, Hamilton SE, Bi K, Schenkel JM, Odumade OA, Casey KA, Thompson EA, Fraser KA, Rosato PC, Filali-Mouhim A, et al (2016) Normalizing the 
environment recapitulates adult human immune traits in laboratory mice. Nature 532: 512-516. doi:10.1038/nature17655

Bieche I, Chavey C, Andrieu C, Busson M, Vacher S, Le Corre L, Guinebretiere JM, Burlinchon S, Lidereau R, Lazennec G (2007) CXC chemokines located in the 4q21 region are up-regulated in breast cancer. Endocr Relat Cancer 14: 1039-1052. doi:10.1677/erc.1.01301

Bloom SM, Bijanki VN, Nava GM, Sun L, Malvin NP, Donermeyer DL, Dunne WM Jr, Allen PM, Stappenbeck TS (2011) Commensal Bacteroides species induce colitis in host-genotype-specific fashion in a mouse model of inflammatory bowel disease. Cell Host Microbe 9: 390-403. doi:10.1016/j.chom.2011.04.009

Boisvert WA, Curtiss LK, Terkeltaub RA (2000) Interleukin-8 and its receptor CXCR2 in atherosclerosis. Immunol Res 21: 129-137. doi:10.1385/ir:21:23:129

Bole-Feysot C, Goffin V, Edery M, Binart N, Kelly PA (1998) Prolactin (PRL) and its receptor: Actions, signal transduction pathways and phenotypes observed in PRL receptor knockout mice. Endocr Rev 19: 225-268. doi:10.1210/edrv.19.3.0334

Brisken C, Heineman A, Chavarria T, Elenbaas B, Tan J, Dey SK, McMahon JA, McMahon AP, Weinberg RA (2000) Essential function of Wnt-4 in mammary gland development downstream of progesterone signaling. Genes Dev 14: 650-654. doi:10.1101/gad.14.6.650

Broxmeyer HE, Cooper S, Cacalano G, Hague NL, Bailish E, Moore MW (1996) Involvement of interleukin (IL) 8 receptor in negative regulation of myeloid progenitor cells in vivo: Evidence from mice lacking the murine IL-8 receptor homologue. J Exp Med 184: 1825-1832. doi:10.1084/jem.184.5.1825

Cacalano G, Lee J, Kikly K, Ryan AM, Pitts-Meek S, Hultgren B, Wood WI, Moore MW (1994) Neutrophil and B cell expansion in mice that lack the murine IL-8 receptor homolog. Science 265: 682-684. doi:10.1126/ science. 8036519

Cadwell K, Liu JY, Brown SL, Miyoshi H, Loh J, Lennerz JK, Kishi C, Kc W, Carrero JA, Hunt S, et al (2008) A key role for autophagy and the autophagy gene Atg16l1 in mouse and human intestinal Paneth cells. Nature 456: 259-263. doi:10.1038/nature07416

Cadwell K, Patel KK, Maloney NS, Liu TC, Ng AC, Storer CE, Head RD, Xavier R, Stappenbeck TS, Virgin HW (2010) Virus-plus-susceptibility gene interaction determines Crohn's disease gene Atg16L1 phenotypes in intestine. Cell 141: 1135-1145. doi:10.1016/j.cell.2010.05.009

Caturegli P, Newschaffer C, Olivi A, Pomper MG, Burger PC, Rose NR (2005) Autoimmune hypophysitis. Endocr Rev 26: 599-614. doi:10.1210/ er.2004-0011

Chapman JC, Polanco JR, Min S, Michael SD (2005) Mitochondrial 3 betahydroxysteroid dehydrogenase (HSD) is essential for the synthesis of progesterone by corpora lutea: An hypothesis. Reprod Biol Endocrinol 3: 11. doi:10.1186/1477-7827-3-11

Chaves I, van der Horst GT, Schellevis R, Nijman RM, Koerkamp MG, Holstege FC, Smidt MP, Hoekman MF (2014) Insulin-FOXO3 signaling modulates circadian rhythms via regulation of clock transcription. Curr Biol 24: 1248-1255. doi:10.1016/j.cub.2014.04.018

Chavey C, Lazennec G, Lagarrigue S, Clape C, Iankova I, Teyssier J, Annicotte JS, Schmidt J, Mataki C, Yamamoto H, et al (2009) CXC ligand 5 is an adipose-tissue derived factor that links obesity to insulin resistance. Cell Metab 9: 339-349. doi:10.1016/ j.cmet.2009.03.002

Chen H, Zhang Y, Dai L, Song Y, Wang Y, Zhou B, Zhou R (2019) Association between polymorphisms in CXCR2 gene and preeclampsia. Mol Genet Genomic Med 7: e00578. doi:10.1002/mgg3.578

Choi JH, Ishida M, Matsuwaki T, Yamanouchi K, Nishihara M (2008) Involvement of 20alpha-hydroxysteroid dehydrogenase in the maintenance of pregnancy in mice. J Reprod Dev 54: 408-412. doi:10.1262/jrd.20045
Ciarloni L, Mallepell S, Brisken C (2007) Amphiregulin is an essential mediator of estrogen receptor alpha function in mammary gland development. Proc Natl Acad Sci U S A 104: 5455-5460. doi:10.1073/pnas.0611647104

Cummings CJ, Martin TR, Frevert CW, Quan JM, Wong VA, Mongovin SM, Hagen TR, Steinberg KP, Goodman RB (1999) Expression and function of the chemokine receptors CXCR1 and CXCR2 in sepsis. I Immunol 162: 2341-2346.

Cypowyj S, Picard C, Marodi L, Casanova JL, Puel A (2012) Immunity to infection in IL-17-deficient mice and humans. Eur J Immunol 42: 2246-2254. doi:10.1002/eji.201242605

Devalaraja RM, Nanney LB, Du J, Qian Q, Yu Y, Devalaraja MN, Richmond A (2000) Delayed wound healing in CXCR2 knockout mice. J Invest Dermatol 115: 234-244. doi:10.1046/j.1523-1747.2000.00034.X

Edgar R, Domrachev M, Lash AE (2002) Gene expression omnibus: NCBI gene expression and hybridization array data repository. Nucleic Acids Res 30: 207-210. doi:10.1093/nar/30.1.207

Escobar P, Bouclier C, Serret J, Bieche I, Brigitte M, Caicedo A, Sanchez E, Vacher S, Vignais ML, Bourin P, et al (2015) IL-1beta produced by aggressive breast cancer cells is one of the factors that dictate their interactions with mesenchymal stem cells through chemokine production. Oncotarget 6: 29034-29047. doi:10.18632/oncotarget.4732

Freund A, Chauveau C, Brouillet JP, Lucas A, Lacroix M, Licznar A, Vignon F, Lazennec $G$ (2003) IL-8 expression and its possible relationship with estrogen-receptor-negative status of breast cancer cells. Oncogene 22: 256-265. doi:10.1038/sj.onc.1206113

Freund A, Jolivel V, Durand S, Kersual N, Chalbos D, Chavey C, Vignon F, Lazennec G (2004) Mechanisms underlying differential expression of interleukin-8 in breast cancer cells. Oncogene 23: 6105-6114. doi:10.1038/sj.onc.1207815

Henrot P, Prevel R, Berger P, Dupin I (2019) Chemokines in COPD: From implication to therapeutic use. Int J Mol Sci 20: E2785. doi:10.3390/ ijms20112785

Hill JM (2007) Vasoactive intestinal peptide in neurodevelopmental disorders: Therapeutic potential. Curr Pharm Des 13: 1079-1089. doi:10.2174/138161207780618975

Honda K, Littman DR (2016) The microbiota in adaptive immune homeostasis and disease. Nature 535: 75-84. doi:10.1038/nature18848

Hooper LV, Littman DR, Macpherson AJ (2012) Interactions between the microbiota and the immune system. Science 336: 1268-1273. doi:10.1126/science. 1223490

Horseman ND, Zhao W, Montecino-Rodriguez E, Tanaka M, Nakashima K, Engle SJ, Smith F, Markoff E, Dorshkind K (1997) Defective mammopoiesis, but normal hematopoiesis, in mice with a targeted disruption of the prolactin gene. EMBO J 16: 6926-6935. doi:10.1093/ emboj/16.23.6926

Howlin J, McBryan J, Martin F (2006) Pubertal mammary gland development Insights from mouse models. I Mammary Gland Biol Neoplasia 11: 283-297. doi:10.1007/s10911-006-9024-2

Hu YC, Wang PH, Yeh S, Wang RS, Xie C, Xu Q, Zhou X, Chao HT, Tsai MY, Chang C (2004) Subfertility and defective folliculogenesis in female mice lacking androgen receptor. Proc Natl Acad Sci U S A 101: 11209-11214. doi:10.1073/pnas.0404372101

Ishida M, Choi JH, Hirabayashi K, Matsuwaki T, Suzuki M, Yamanouchi K, Horai R, Sudo K, Iwakura Y, Nishihara M (2007) Reproductive phenotypes in mice with targeted disruption of the 20alpha-hydroxysteroid dehydrogenase gene. J Reprod Dev 53: 499-508. doi:10.1262/jrd.18125

Jarde T, Dale T (2012) Wnt signalling in murine postnatal mammary gland development. Acta Physiol 204: 118-127. doi:10.1111/j.17481716.2011.02283.x

Kang X, Zhang X, Liu Z, Xu H, Wang T, He L, Zhao A (2016) CXCR2-mediated granulocytic myeloid-derived suppressor cells' functional 
characterization and their role in maternal fetal interface. DNA Cell Biol 35: 358-365. doi:10.1089/dna.2015.2962

Kelly PA, Bachelot A, Kedzia C, Hennighausen L, Ormandy CJ, Kopchick JJ, Binart N (2002) The role of prolactin and growth hormone in mammary gland development. Mol Cell Endocrinol 197: 127-131. doi:10.1016/s0303-7207(02)00286-1

Ketchesin KD, Stinnett GS, Seasholtz AF (2017) Corticotropin-releasing hormone-binding protein and stress: From invertebrates to humans. Stress 20: 449-464. doi:10.1080/10253890.2017.1322575

Kouros-Mehr H, Slorach EM, Sternlicht MD, Werb Z (2006) GATA-3 maintains the differentiation of the luminal cell fate in the mammary gland. Cell 127: 1041-1055. doi:10.1016/j.cell.2006.09.048

Lazennec G, Lam PY (2016) Recent discoveries concerning the tumor: Mesenchymal stem cell interactions. Biochim Biophys Acta 1866: 290-299. doi:10.1016/j.bbcan.2016.10.004

Lazennec G, Richmond A (2010) Chemokines and chemokine receptors: New insights into cancer-related inflammation. Trends Mol Med 16: 133-144. doi:10.1016/j.molmed.2010.01.003

Li JJ, Ma FX, Wang YW, Chen F, Lu SH, Chi Y, Du WJ, Song BQ, Hu LD, Chen H, et al (2017) Knockdown of IL-8 provoked premature senescence of placenta-derived mesenchymal stem cells. Stem Cells Dev 26: 912-931. doi:10.1089/scd.2016.0324

Liu L, Belkadi A, Darnall L, Hu T, Drescher C, Cotleur AC, Padovani-Claudio D, He T, Choi K, Lane TE, et al (2010) CXCR2-positive neutrophils are essential for cuprizone-induced demyelination: Relevance to multiple sclerosis. Nat Neurosci 13: 319-326. doi:10.1038/nn.2491

Loh R, Chau L, Aijaz A, Wu K, Galvez R (2017) Antagonizing the different stages of kappa opioid receptor activation selectively and independently attenuates acquisition and consolidation of associative memories. Behav Brain Res 323: 1-10. doi:10.1016/j.bbr.2017.01.032

Luetteke NC, Qiu TH, Fenton SE, Troyer KL, Riedel RF, Chang A, Lee DC (1999) Targeted inactivation of the EGF and amphiregulin genes reveals distinct roles for EGF receptor ligands in mouse mammary gland development. Development 126: 2739-2750.

Markus MA, Morris BJ (2009) RBM4: A multifunctional RNA-binding protein. Int J Biochem Cell Biol 41: 740-743. doi:10.1016/j.biocel.2008.05.027

McCafferty J, Muhlbauer M, Gharaibeh RZ, Arthur JC, Perez-Chanona E, Sha W, Jobin C, Fodor AA (2013) Stochastic changes over time and not founder effects drive cage effects in microbial community assembly in a mouse model. ISME J 7: 2116-2125. doi:10.1038/ismej.2013.106

McCully ML, Kouzeli A, Moser B (2018) Peripheral tissue chemokines: Homeostatic control of immune surveillance T cells. Trends Immunol 39: 734-747. doi:10.1016/j.it.2018.06.003

Nirmal AJ, Regan T, Shih BB, Hume DA, Sims AH, Freeman TC (2018) Immune cell gene signatures for profiling the microenvironment of solid tumors. Cancer Immunol Res 6: 1388-1400. doi:10.1158/2326-6066.cir18-0342

Okada H, Kuhn C, Feillet H, Bach JF (2010) The "hygiene hypothesis" for autoimmune and allergic diseases: An update. Clin Exp Immunol 160: 1-9. doi:10.1111/j.1365-2249.2010.04139.x

Payne AH, Clarke TR, Bain PA (1995) The murine 3 beta-hydroxysteroid dehydrogenase multigene family: Structure, function and tissuespecific expression. J Steroid Biochem Mol Biol 53: 111-118. doi:10.1016/ 0960-0760(95)00028-x

Piekorz RP, Gingras S, Hoffmeyer A, Ihle JN, Weinstein Y (2005) Regulation of progesterone levels during pregnancy and parturition by signal transducer and activator of transcription 5 and 20alphahydroxysteroid dehydrogenase. Mol Endocrinol 19: 431-440. doi:10.1210/me.2004-0302

Pritchett-Corning KR, Cosentino J, Clifford CB (2009) Contemporary prevalence of infectious agents in laboratory mice and rats. Lab Anim 43: 165-173. doi:10.1258/la.2008.008009
Ramanan D, Tang MS, Bowcutt R, Loke P, Cadwell K (2014) Bacterial sensor Nod2 prevents inflammation of the small intestine by restricting the expansion of the commensal Bacteroides vulgatus. Immunity 41: 311-324. doi:10.1016/j.immuni.2014.06.015

Ramanan D, Bowcutt R, Lee SC, Tang MS, Kurtz ZD, Ding Y, Honda K, Gause WC Blaser MJ, Bonneau RA, et al (2016) Helminth infection promotes colonization resistance via type 2 immunity. Science 352: 608-612. doi:10.1126/science.aaf3229

Reese TA, Bi K, Kambal A, Filali-Mouhim A, Beura LK, Burger MC, Pulendran B, Sekaly RP, Jameson SC, Masopust D, et al (2016) Sequential infection with common pathogens promotes human-like immune gene expression and altered vaccine response. Cell Host Microbe 19: 713-719. doi:10.1016/j.chom.2016.04.003

Rexach JE, Clark PM, Mason DE, Neve RL, Peters EC, Hsieh-Wilson LC (2012) Dynamic O-GlcNAC modification regulates CREB-mediated gene expression and memory formation. Nat Chem Biol 8: 253-261. doi:10.1038/nchembio.770

Rosenfeld CS, Roberts RM, Lubahn DB (2001) Estrogen receptor- and aromatase-deficient mice provide insight into the roles of estrogen within the ovary and uterus. Mol Reprod Dev 59: 336-346. doi:10.1002/ mrd.1039

Rosshart SP, Herz J, Vassallo BG, Hunter A, Wall MK, Badger JH, McCulloch JA, Anastasakis DG, Sarshad AA, Leonardi I, et al (2019) Laboratory mice born to wild mice have natural microbiota and model human immune responses. Science 365: eaaw4361. doi:10.1126/science.aaw4361

Roy S, Trinchieri G (2017) Microbiota: A key orchestrator of cancer therapy. Nat Rev Cancer 17: 271-285. doi:10.1038/nrc.2017.13

Scher JU, Sczesnak A, Longman RS, Segata N, Ubeda C, Bielski C, Rostron T Cerundolo V, Pamer EG, Abramson SB, et al (2013) Expansion of intestinal Prevotella copri correlates with enhanced susceptibility to arthritis. Elife 2: e01202. doi:10.7554/elife.01202

Shiina H, Matsumoto T, Sato T, Igarashi K, Miyamoto J, Takemasa S, Sakari M, Takada I, Nakamura T, Metzger D, et al (2006) Premature ovarian failure in androgen receptor-deficient mice. Proc Natl Acad Sci U S A 103: 224-229. doi:10.1073/pnas.0506736102

Stappenbeck TS, Virgin HW (2016) Accounting for reciprocal host-microbiome interactions in experimental science. Nature 534:191-199. doi:10.1038/ nature18285

Stocco C, Telleria C, Gibori G (2007) The molecular control of corpus luteum formation, function, and regression. Endocr Rev 28: 117-149. doi:10.1210/er.2006-0022

Strieter RM, Burdick MD, Gomperts BN, Belperio JA, Keane MP (2005a) CXC chemokines in angiogenesis. Cytokine Growth Factor Rev 16: 593-609. doi:10.1016/j.cytogfr.2005.04.007

Strieter RM, Keane MP, Burdick MD, Sakkour A, Murray LA, Belperio JA (2005b) The role of CXCR2/CXCR2 ligands in acute lung injury. Curr Drug Targets Inflamm Allergy 4: 299-303. doi:10.2174/1568010054022178

Sturn A, Quackenbush J, Trajanoski Z (2002) Genesis: Cluster analysis of microarray data. Bioinformatics 18: 207-208. doi:10.1093/ bioinformatics/18.1.207

Tao L, Reese TA (2017) Making mouse models that reflect human immune responses. Trends Immunol 38: 181-193. doi:10.1016/j.it.2016.12.007

Thorburn AN, McKenzie Cl, Shen S, Stanley D, Macia L, Mason LJ, Roberts LK, Wong CH, Shim R, Robert R, et al (2015) Evidence that asthma is a developmental origin disease influenced by maternal diet and bacterial metabolites. Nat Commun 6: 7320. doi:10.1038/ncomms8320

Toda K, Takeda K, Okada T, Akira S, Saibara T, Kaname T, Yamamura K, Onish S, Shizuta $Y$ (2001) Targeted disruption of the aromatase $\mathrm{P} 450$ gene (Cyp19) in mice and their ovarian and uterine responses to 17betaoestradiol. J Endocrinol 170: 99-111. doi:10.1677/joe.0.1700099

Tsai HH, Frost E, To V, Robinson S, Ffrench-Constant C, Geertman R, Ransohoff RM, Miller RH (2002) The chemokine receptor CXCR2 controls 
positioning of oligodendrocyte precursors in developing spinal cord by arresting their migration. Cell 110: 373-383. doi:10.1016/s00928674(02)00838-3

Walters KA (2015) Role of androgens in normal and pathological ovarian function. Reproduction 149: R193-R218. doi:10.1530/rep-14-0517

Wang J, Knaut H (2014) Chemokine signaling in development and disease. Development 141: 4199-4205. doi:10.1242/dev.101071

Wang Z, Roberts AB, Buffa JA, Levison BS, Zhu W, Org E, Gu X, Huang Y, Zamanian-Daryoush M, Culley MK, et al (2015) Non-lethal inhibition of gut microbial trimethylamine production for the treatment of atherosclerosis. Cell 163: 1585-1595. doi:10.1016/j.cell.2015.11.055

Watanabe K, Gilchrist CA, Uddin MJ, Burgess SL, Abhyankar MM, Moonah SN, Noor Z, Donowitz JR, Schneider BN, Arju T, et al (2017) Microbiomemediated neutrophil recruitment via CXCR2 and protection from amebic colitis. PLoS Pathog 13: e1006513. doi:10.1371/ journal.ppat.1006513

Watson CJ, Khaled WT (2008) Mammary development in the embryo and adult: A journey of morphogenesis and commitment. Development 135: 995-1003. doi:10.1242/dev.005439
Wu D, Hong H, Huang X, Huang L, He Z, Fang Q, Luo Y (2016) CXCR2 is decreased in preeclamptic placentas and promotes human trophoblast invasion through the Akt signaling pathway. Placenta 43: 17-25. doi:10.1016/ j.placenta.2016.04.016

Yanaihara A, Mitsukawa K, Iwasaki S, Otsuki K, Kawamura T, Okai T (2007) High concentrations of lactoferrin in the follicular fluid correlate with embryo quality during in vitro fertilization cycles. Fertil Steril 87: 279-282. doi:10.1016/j.fertnstert.2006.06.025

Zhou J, Chehab R, Tkalcevic J, Naylor MJ, Harris J, Wilson TJ, Tsao S, Tellis I, Zavarsek S, Xu D, et al (2005) Elf5 is essential for early embryogenesis and mammary gland development during pregnancy and lactation. EMBO J 24: 635-644. doi:10.1038/sj.emboj.7600538

Zlotnik A, Yoshie $O$ (2000) Chemokines: A new classification system and their role in immunity. Immunity 12: 121-127. doi:10.1016/s1074-7613(00)80165-X

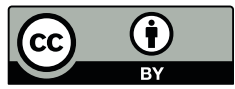

License: This article is available under a Creative Commons License (Attribution 4.0 International, as described at https://creativecommons.org/ licenses/by/4.0/). 